\title{
Efficient estimation of the error distribution function in heteroskedastic nonparametric regression with missing data
}

\author{
Justin Chown
}

\begin{abstract}
A residual-based empirical distribution function is proposed to estimate the distribution function of the errors of a heteroskedastic nonparametric regression with responses missing at random based on completely observed data, and this estimator is shown to be asymptotically most precise.
\end{abstract}

keywords: efficient estimator, empirical distribution function, heteroskedasticity, local polynomial smoother, nonparametric regression, transfer principle

2010 AMS Subject Classifications: Primary: 62G05; Secondary: 62G08, 62G20

\section{Introduction}

An important problem encountered in practice occurs when variation in the data is found to be dynamic. A typical example is when responses $Y$ are regressed onto a vector of $m$ covariates $X$ and the errors of that regression have variation changing in $X$. Under this condition, many statistical procedures no longer provide consistent inference. For example, consider a study of crop yields under different application amounts of a fertilizer. When the variation in yields depends on the amount of fertilizer applied, the classical F-test will no longer provide a consistent method of inference for a regression of these crop yields toward the amount of fertilizer applied because it assumes the model errors have constant variation. Examples of heteroskedastic data may be found in Greene (2000), Vinod (2008), Sheather (2009) and Asteriou and Hall (2011).

We are interested in the case where the responses are missing. This means observing a random sample $\left(X_{1}, \delta_{1} Y_{1}, \delta_{1}\right), \ldots,\left(X_{n}, \delta_{n} Y_{n}, \delta_{n}\right)$ of data that is composed of independent and identically distributed copies of a base observation $(X, \delta Y, \delta)$. Here $\delta$ is an indicator variable taking values one, when $Y$ is observed, and zero, otherwise. Throughout this article, we will interpret a datum $(X, 0,0)$ as corresponding to a categorically missing response, i.e. when $\delta=0$, the first zero in the datum only describes the product $0 \times Y=0$, almost surely, because we make the common assumption that $P(|Y|<\infty)=1$. For this work, we make the following common assumption, which ensures good performance of the nonparametric function estimators studied in this article, concerning the covariates $X$ :

Assumption 1. The covariate vector $X$ has a distribution that is quasi-uniform on the cube $[0,1]^{m}$; i.e. $X$ has a density that is both bounded and bounded away from zero on $[0,1]^{m}$.

We assume the responses are missing at random (MAR), and, paraphrasing Chown and Müller (2013), we will refer to the probability model with responses missing at random as the MAR model. This means the distribution of $\delta$ given both the covariates $X$ and the response $Y$ depends only on the covariates $X$, i.e.

$$
P(\delta=1 \mid X, Y)=P(\delta=1 \mid X)=\pi(X) .
$$

Ruhr-Universität Bochum, Fakultät für Mathematik, Lehrstuhl für Stochastik, 44780 Bochum, DE

Email: justin.chown@ruhr-uni-bochum.de. 
Since we do observe some responses $Y$, we will assume that $\pi$ is almost everywhere bounded away from zero on $[0,1]^{m}$. It is then clear that $E \delta=E[\pi(X)]$ is positive. The MAR assumption is commonly used and it is very reasonable in many missing data situations (see Chapter 1 of Little and Rubin, 2002).

In this article we study the heteroskedastic nonparametric regression model

$$
Y=r(X)+\sigma(X) e,
$$

with the error $e$ independent of the covariate vector $X$. In order to identify the functions $r$ and $\sigma$, we will additionally assume the error $e$ has mean zero and unit variance. For this work, we are interested in the case of smooth functions $r$ and $\sigma$ (see below for an explicit definition), and we will assume that $\sigma$ is a positive-valued function so that it is a well-defined scale function. Hence, the model above is a well-defined heteroskedastic nonparametric regression model with identifiable components. This model is closely related to that studied in Chown and Müller (2013), who study the simpler case of $\sigma(\cdot) \equiv \sigma_{0}$, a positive constant, i.e. $\sigma(x)=\sigma_{0}$ for almost every $x$. Consequently, many results will be familiar. Here we will need to estimate the two unknown functions $r$ and $\sigma$ with nonparametric function estimators that are constructed from the assumed smoothness properties of these functions. We will then use these estimates in the proposed estimator of the distribution function of the errors $F$.

To begin, we first consider (1.1) and observe that

$$
E[\delta h(e)]=E \delta E[h(e)] \quad \text { and } \quad E[\delta h(e) \mid X]=\pi(X) E[h(e)]
$$

for suitable measurable functions $h$. The relations above naturally lead to complete case estimators for each of $F, r$ and $\sigma$. We investigate the residual-based empirical distribution function, $\hat{\mathbb{F}}_{c}$, given as

$$
\hat{\mathbb{F}}_{c}(t)=\frac{1}{N} \sum_{j=1}^{n} \delta_{j} \mathbf{1}\left[\hat{\varepsilon}_{j, c} \leq t\right]=\frac{1}{N} \sum_{j=1}^{n} \delta_{j} \mathbf{1}\left[\frac{Y_{j}-\hat{r}_{c}\left(X_{j}\right)}{\hat{\sigma}_{c}\left(X_{j}\right)} \leq t\right], \quad t \in \mathbb{R} .
$$

Here $N=\sum_{j=1}^{n} \delta_{j}$ is the number of completely observed pairs $(X, Y)$ and the subscript "c" indicates the estimator is based on the subsample of complete cases described below, which is, in general, different from the original sample of data. Similar to the estimator of Chown and Müller (2013), this is a complete case estimator.

To better explain the idea, first consider the sample $\left(X_{1}, \delta_{1} Y_{1}, \delta_{1}\right), \ldots,\left(X_{n}, \delta_{n} Y_{n}, \delta_{n}\right)$. Due to the i.i.d. nature of the this sample, we can order it in any configuration such that triples with $\delta_{j}=1$ are listed before triples with $\delta_{j}=0$. This means we can write the sample as two subsamples: $\left(X_{1}, Y_{1}, 1\right), \ldots,\left(X_{N}, Y_{N}, 1\right)$ and $\left(X_{N+1}, 0,0\right), \ldots,\left(X_{n}, 0,0\right)$. The first subsample is called the complete cases and $N \leq n$ is the random size of the complete cases. Hence, the estimators studied in this article use only the part of the original sample where the responses $Y_{j}$ are actually observed. For the estimator $\hat{\mathbb{F}}_{c}$ given in (1.3), this means we use only the residuals that can actually be constructed directly from the observed responses:

$$
\hat{\varepsilon}_{j, c}=\frac{Y_{j}-\hat{r}_{c}\left(X_{j}\right)}{\hat{\sigma}_{c}\left(X_{j}\right)}, \quad j=1, \ldots, N
$$

where $\hat{r}_{c}$ and $\hat{\sigma}_{c}$ are each respectively suitable complete case estimators of the regression function $r$ and the scale function $\sigma$. Since we are only using a part of the original data based on the auxiliary information that $\delta=1$, which now has different stochastic properties than the original data, we will, nevertheless, argue below that $\hat{\mathbb{F}}_{c}$ is both a consistent and an efficient estimator for $F$.

In this work, we use local polynomial estimators of the first and second conditional moments $r(x)=E(Y \mid X=x)$ and $r_{2}(x)=E\left(Y^{2} \mid X=x\right)$, respectively, which we will use later to construct our estimators $\hat{r}_{c}$ and $\hat{\sigma}_{c}$. Local polynomial estimation follows naturally by a Taylor expansion argument, and, therefore, follows from both of the functions $r$ and $\sigma$ satisfying certain smoothness conditions; i.e. we assume both $r$ and $\sigma$ lie on the Hölder space of functions 
$H(d, \varphi)$ with domain $[0,1]^{m}$. Paraphrasing Müller, Schick and Wefelmeyer (2009), a function from $[0,1]^{m}$ to $\mathbb{R}$ belongs to $H(d, \varphi)$, if it has continuous partial derivatives up to order $d$ and the partial derivatives of order $d$ are Hölder with exponent $0<\varphi \leq 1$. We will write $H_{1}(d, \varphi)$ for the unit ball of $H(d, \varphi)$ (see Müller, Schick and Wefelmeyer, 2009, for an explicit definition).

To define the local polynomial estimators of degree $d$, we first introduce some notation. Let $I(d)$ be the set of multi-indices $i=\left(i_{1}, \ldots, i_{m}\right)$ such that $i_{1}+\cdots+i_{m} \leq d$. These multi-indices correspond with the partial derivatives of $r$ and $r_{2}$ (and hence $\sigma$ ) whose order is at most $d$. The local polynomial estimators of $r$ and $r_{2}$ are respectively given by $\hat{\gamma}_{a, 0}$, for $a=1,2$, where $\hat{\gamma}_{a, 0}$ denotes the $0=(0, \ldots, 0)$ entry of the vector

$$
\hat{\gamma}_{a}=\underset{\gamma=\left(\gamma_{i}\right)_{i \in I(d)}}{\arg \min } \sum_{j=1}^{n} \delta_{j}\left\{Y_{j}^{a}-\sum_{i \in I(d)} \gamma_{i} \psi_{i}\left(\frac{X_{j}-x}{\lambda_{n}}\right)\right\}^{2} w\left(\frac{X_{j}-x}{\lambda_{n}}\right), \quad a=1,2 .
$$

Here

$$
\psi_{i}(x)=\frac{x_{1}^{i_{1}}}{i_{1} !} \cdots \frac{x_{m}^{i_{m}}}{i_{m} !}, \quad x=\left(x_{1}, \ldots, x_{m}\right) \in[0,1]^{m},
$$

$w(x)=w_{1}\left(x_{1}\right) \cdots w_{m}\left(x_{m}\right)$ is a product of densities, and $\left\{\lambda_{n}\right\}_{n \geq 1}$ is a bandwidth sequence, i.e. a sequence of positive numbers satisfying $\lambda_{n} \rightarrow 0$, as $n \rightarrow \infty$. Hence, we introduce our respective function estimators of $r$ and $\sigma$ pointwise at each $x \in[0,1]^{m}$ as $\hat{r}_{c}(x)=\hat{\gamma}_{1,0}$ and $\hat{\sigma}_{c}(x)=\left\{\hat{\gamma}_{2,0}-\hat{\gamma}_{1,0}^{2}\right\}^{1 / 2}$. Note that $\delta_{1}, \ldots, \delta_{n}$ appear in the formula above because we require only the complete cases to estimate both $r$ and $r_{2}$, and the minimization procedure above is unaffected by the proportion $\pi$ of missing data.

Neumeyer and Van Keilegom (2010) construct an estimator related to $\hat{\mathbb{F}}_{c}$ for the full model using local polynomial estimators of the first and second conditional moments, i.e. the simpler case where $\delta_{j}=1, j=1, \ldots, n$. However, these authors require the density function of the covariates $g$ to be differentiable. For our model, we work with the conditional density function $g_{1}$ of the covariates $X$ given $\delta=1$, and this differentiability requirement would then be imposed on $g_{1}$. Using the identity for the conditional distribution function $G_{1}$ of the covariates $X$ given $\delta=1$, we have $G_{1}(d x)=\{\pi(x) / E \delta\} G(d x)$, and we can see that any differentiability requirements imposed on the density function $g_{1}$ must also apply to $\pi$.

To alleviate this differentiability requirement, we turn our attention to the results of Müller, Schick and Wefelmeyer (2007, 2009), who impose no such requirement. Investigating the proof of Lemma 1 of Müller, Schick and Wefelmeyer (2009), reveals straightforward modifications of those results for local polynomial function estimation in a homoskedastic model to the heteroskedastic model considered here. Since this approach requires Assumption 1, using the relation between $G_{1}$ and $G$ above and the bounding assumption on $\pi$, we observe that $G_{1}$ is quasi-uniform whenever $G$ is quasi-uniform. We arrive at the following crucial technical corollary to Lemma 1 of Müller, Schick and Wefelmeyer (2009) for the estimators $\hat{r}_{c}$ and $\hat{\sigma}_{c}$.

Corollary 1. Let Assumption 1 hold. Suppose the regression function $r$ and the scale function $\sigma$ both belong to $H(d, \varphi)$ with domain $[0,1]^{m}$. Further suppose the error variable has mean equal to zero, variance equal to one and a finite moment of order $q>4 s /(2 s-m)$, with $s=d+\varphi>3 m / 2$. Assume the missingness proportion $\pi$ is almost everywhere bounded away from zero on $[0,1]^{m}$, and the densities $w_{1}, \ldots, w_{m}$ are $(m+2)$-times continuously differentiable and have compact support $[-1,1]$. Let $\lambda_{n} \sim(n \log (n))^{-1 /(2 s)}$. Then there is a random function $\hat{a}_{1, c}$, associated to the complete case local polynomial estimate $\hat{r}_{c}$ of $r$, such that

$$
P\left(\hat{a}_{1, c} \in H_{1}(m, \alpha)\right) \rightarrow 1
$$

for some $\alpha>0$,

$$
\int_{[0,1]^{m}}\left|\hat{a}_{1, c}(x)\right|^{1+b} g_{1}(x) d x=o_{p}\left(n^{-1 / 2}\right),
$$


for $b>m /(2 s-m)$, and

$$
\sup _{x \in[0,1]^{m}}\left|\hat{r}_{c}(x)-r(x)-\hat{a}_{1, c}(x)\right|=o_{p}\left(n^{-1 / 2}\right) .
$$

If, additionally, the error variable has a finite moment of order $2 q$, then there is a random function $\hat{a}_{2, c}$, associated to the complete case local polynomial estimate $\hat{r}_{2, c}$ of $r_{2}$, such that

and

$$
\begin{aligned}
& P\left(\hat{a}_{2, c} \in H_{1}(m, \alpha)\right) \rightarrow 1, \\
& \int_{[0,1]^{m}}\left|\hat{a}_{2, c}(x)\right|^{1+b} g_{1}(x) d x=o_{p}\left(n^{-1 / 2}\right)
\end{aligned}
$$

$$
\sup _{x \in[0,1]^{m}}\left|\hat{r}_{2, c}(x)-r_{2}(x)-\hat{a}_{2, c}(x)\right|=o_{p}\left(n^{-1 / 2}\right) .
$$

Paraphrasing Remark 5 of Müller, Schick and Wefelmeyer (2009), there is a trade-off between the required smoothness of the regression and scale functions (indicated by the variable $s$ ) and the existence of higher order moments for the error variable $e$ (indicated by $q$ ). This means that higher degree polynomials, used to approximate $r$ and $\sigma$, require higher order moments of $e$ to exist. Further, we can see that a larger bandwidth may be used to estimate these functions when they are smooth but a smaller bandwidth will be required when these functions are rough. In light of the above results, we are able to obtain analogous conclusions to those of Lemma A.2 of Neumeyer and Van Keilegom (2010).

Proposition 1. Suppose the first set of assumptions of Corollary 1 are satisfied. Then we have

$$
\int_{[0,1]^{m}} \frac{\hat{r}_{c}(x)-r(x)}{\sigma(x)} g_{1}(x) d x=\frac{1}{N} \sum_{j=1}^{n} \delta_{j} e_{j}+o_{p}\left(n^{-1 / 2}\right) .
$$

Now suppose the additional assumptions of Corollary 1 are satisfied. Then we have

$$
\int_{[0,1]^{m}} \frac{\hat{\sigma}_{c}(x)-\sigma(x)}{\sigma(x)} g_{1}(x) d x=\frac{1}{N} \sum_{j=1}^{n} \delta_{j} \frac{e_{j}^{2}-1}{2}+o_{p}\left(n^{-1 / 2}\right) .
$$

REMARK 1. Analogous results to Corollary 1 above hold for the full model where $\delta_{j}=1$, $j=1, \ldots, n$, and $N=n$, almost surely, in both cases where the covariate distribution is $G$ and $G_{1}$. Here the local polynomial estimators $\hat{r}$ for $r$ (with associated $\hat{a}_{1}$ ) and $\hat{r}_{2}$ for $r_{2}$ (with associated $\hat{a}_{2}$ ) are respectively defined exactly as $\hat{r}_{c}$ and $\hat{r}_{2, c}$ are defined above, but now the indicators $\delta_{1}, \ldots, \delta_{n}$ are all equal to one. Hence, we obtain estimators $\hat{r}$ for $r$ and $\hat{\sigma}$ for $\sigma$ for which analogous results of Proposition 1 hold in the full model in both cases where the covariate distribution is $G$ and $G_{1}$. The case of covariates having distribution $G$ confirms the findings of Lemma A.2 of Neumeyer and Van Keilegom (2010), which are required to prove their main result.

As noted in Remark 1 of Chown and Müller (2013), one proves the above statements analogously to how Müller, Schick and Wefelmeyer (2009) prove their results (inspect the proof of Lemma 1 of that paper). The only changes occur by introducing $\sigma$ and the indicators $\delta_{1}, \ldots, \delta_{n}$. Since we also estimate $r_{2}$, this requires strengthening the moment conditions on the error variable $e$ from $q$ to $2 q$ because $Y^{2}=r^{2}(X)+\sigma^{2}(X)+2 r(X) \sigma(X) e+\sigma^{2}(X)\left(e^{2}-1\right)$, which follows from the model equation above. An additional requirement needed by Neumeyer and Van Keilegom (2010) for their results to hold is that $\sup _{t \in \mathbb{R}}\left|t^{2} F^{\prime \prime}(t)\right|<\infty$. This assumption implies the curvature of the function space underlying the probability model is finite. However, we can measure this curvature using Fisher information. This means we can merely assume that $F$ has finite Fisher information for both location and scale, written as Assumption 2 below, which is a lighter assumption than $\sup _{t \in \mathbb{R}}\left|t^{2} F^{\prime \prime}(t)\right|<\infty$. We now arrive at our third auxiliary result, which confirms the results of Neumeyer and Van Keilegom (2010). The proof of this result is rather elaborate and technical. Therefore, it is held to Section 4 . 
Assumption 2. The error density $f$ is absolutely continuous with almost everywhere derivative $f^{\prime}$ and finite Fisher information for both location and scale; i.e.

$$
\int_{-\infty}^{\infty}\left\{1+z^{2}\right\}\left\{\frac{f^{\prime}(z)}{f(z)}\right\}^{2} F(d z)<\infty .
$$

TheOREM 1 (EXPANSION FOR THE FUll MODEL). Assume the covariates $X$ are distributed according to $G$. Let the required assumptions of Proposition 1 be satisfied concerning the local polynomial estimators $\hat{r}$ and $\hat{r}_{2}$ (see Remark 1 above). Further, let Assumption 0 hold with the error density $f$ additionally satisfying $\sup _{t \in \mathbb{R}} f(t)<\infty$ and $\sup _{t \in \mathbb{R}}|t f(t)|<\infty$. Then, for $\hat{\varepsilon}_{1}=\left\{Y_{1}-\hat{r}\left(X_{1}\right)\right\} / \hat{\sigma}\left(X_{1}\right), \ldots, \hat{\varepsilon}_{n}=\left\{Y_{n}-\hat{r}\left(X_{n}\right)\right\} / \hat{\sigma}\left(X_{n}\right)$, we have

$$
\sup _{t \in \mathbb{R}}\left|\frac{1}{n} \sum_{j=1}^{n}\left[\mathbf{1}\left[\hat{\varepsilon}_{j} \leq t\right]-\mathbf{1}\left[e_{j} \leq t\right]-f(t)\left\{e_{j}+\frac{t}{2}\left(e_{j}^{2}-1\right)\right\}\right]\right|=o_{p}\left(n^{-1 / 2}\right) .
$$

We now adapt the results of Theorem 1 to the MAR model using the transfer principle for complete case statistics given in Koul, Müller and Schick (2012). Expanding on the observations of Chown and Müller (2013), it follows that we can factor the joint distribution of $(X, Y)$ into two components: the distribution $G$ of the covariates $X$ and the conditional distribution of the responses $Y$ given $X$, i.e. the distribution $F$ of the errors $e$. Now, using the MAR assumption, we observe that $Y$ and $\delta$ are independent given $X$. This implies only the distribution $G$ changes to $G_{1}$ when moving from full model to the MAR model, e.g. complete case statistics are based on observations $(X, Y)$ with a joint conditional distribution given $\delta=1$, which can now be factored into $G_{1}$ and $F$. Hence, the functionals $F, r$ and $\sigma$ remain the same in the MAR model. This implies the complete case statistic $\hat{\mathbb{F}}_{c}$ is a consistent estimator for $F$ in the MAR model. However, in order to apply the transfer principle, we need to restate the result of Theorem 1 for covariates that have distribution $G_{1}$, which corresponds to the data used in our complete case estimator $\hat{\mathbb{F}}_{c}$. The proof of this result follows immediately from the proof of Theorem 1 (see Section 4) with the discussion in Remark 1 above.

Corollary 2 (EXPANSION FOR The FUll MODEL USING $G_{1}$ ). Assume the covariates $X$ are distributed according to $G_{1}$, and $\pi$ is almost everywhere bounded away from zero on $[0,1]^{m}$. Let the required assumptions of Proposition 1 be satisfied concerning the local polynomial estimators $\hat{r}$ and $\hat{r}_{2}$ (see Remark 1 above). Further, let Assumption 0 hold with the error density $f$ additionally satisfying $\sup _{t \in \mathbb{R}} f(t)<\infty$ and $\sup _{t \in \mathbb{R}}|t f(t)|<\infty$. Then, for $\hat{\varepsilon}_{1}=$ $\left\{Y_{1}-\hat{r}\left(X_{1}\right)\right\} / \hat{\sigma}\left(X_{1}\right), \ldots, \hat{\varepsilon}_{n}=\left\{Y_{n}-\hat{r}\left(X_{n}\right)\right\} / \hat{\sigma}\left(X_{n}\right)$, we have

$$
\sup _{t \in \mathbb{R}}\left|\frac{1}{n} \sum_{j=1}^{n}\left[\mathbf{1}\left[\hat{\varepsilon}_{j} \leq t\right]-\mathbf{1}\left[e_{j} \leq t\right]-f(t)\left\{e_{j}+\frac{t}{2}\left(e_{j}^{2}-1\right)\right\}\right]\right|=o_{p}\left(n^{-1 / 2}\right) .
$$

Combining the results above with the transfer principle for complete case statistics, we can immediately derive the expansion of our complete case estimator $\hat{\mathbb{F}}_{c}$. We investigate the efficiency bound for regular estimators of $F$ in the MAR model in Section 2 below, i.e. estimators whose limit distributions do not depend on any direction of approach. In Corollary 3 (see Section 2 below), we provide the efficient influence function characterizing the class of efficient estimators of $F$ in the MAR model. Since the influence function of our complete case estimator $\hat{\mathbb{F}}_{c}$ matches the efficient influence function, this characterizes $\hat{\mathbb{F}}_{c}$ as an efficient estimator for $F$ in the MAR model, which implies that $\hat{\mathbb{F}}_{c}$ is an asymptotically most precise (least dispersed) estimator. We now arrive at the main result of this section:

TheOREM 2 (EXPANSION FOR THE MAR MODEL). Consider the heteroskedastic nonparametric regression model with responses missing at random. Let Assumption 0 hold with the error density $f$ additionally satisfying $\sup _{t \in \mathbb{R}} f(t)<\infty$ and $\sup _{t \in \mathbb{R}}|t f(t)|<\infty$, and let the assumptions of Corollary 1 hold. Then the complete case estimator $\hat{\mathbb{F}}_{c}$ of the error distribution 
function $F$ satisfies the uniform stochastic expansion

$$
\sup _{t \in \mathbb{R}}\left|\hat{\mathbb{F}}_{c}-\frac{1}{N} \sum_{j=1}^{n} \delta_{j} \mathbf{1}\left[e_{j} \leq t\right]-\frac{1}{N} \sum_{j=1}^{n} \delta_{j} f(t)\left\{e_{j}+\frac{t}{2}\left(e_{j}^{2}-1\right)\right\}\right|=o_{p}\left(n^{-1 / 2}\right) .
$$

Furthermore, $\hat{\mathbb{F}}_{c}$ is asymptotically linear, uniformly in $t \in \mathbb{R}$, with influence function

$$
\phi(\delta, e, t)=\frac{\delta}{E \delta}\left[\mathbf{1}[e \leq t]-F(t)+f(t)\left\{e+\frac{t}{2}\left(e^{2}-1\right)\right\}\right]
$$

and $\hat{\mathbb{F}}_{c}$ is asymptotically efficient, in the sense of Hájek and Le Cam, for estimating F.

Proof. The assumptions of Theorem 1 and Corollary 2 are satisfied. Hence, for the full model, we have

$$
\sup _{t \in \mathbb{R}}\left|\frac{1}{n} \sum_{j=1}^{n}\left[\mathbf{1}\left[\hat{\varepsilon}_{j} \leq t\right]-\mathbf{1}\left[e_{j} \leq t\right]-f(t)\left\{e_{j}+\frac{t}{2}\left(e_{j}^{2}-1\right)\right\}\right]\right|=o_{p}\left(n^{-1 / 2}\right),
$$

when the covariates $X$ are distributed under either $G$ or $G_{1}$. Since $\hat{\mathbb{F}}_{c}$ is the complete case version of the estimator in the display above, it follows from Remark 2.5 of Koul, Müller and Schick (2012) for the first assertion to hold, i.e.

$$
\sup _{t \in \mathbb{R}}\left|\hat{\mathbb{F}}_{c}-\frac{1}{N} \sum_{j=1}^{n} \delta_{j} \mathbf{1}\left[e_{j} \leq t\right]-\frac{1}{N} \sum_{j=1}^{n} \delta_{j} f(t)\left\{e_{j}+\frac{t}{2}\left(e_{j}^{2}-1\right)\right\}\right|=o_{p}\left(n^{-1 / 2}\right) .
$$

This expansion is equivalent to

$$
\sup _{t \in \mathbb{R}}\left|\frac{1}{n} \sum_{j=1}^{n} \frac{\delta_{j}}{E \delta}\left[\mathbf{1}\left[\hat{\varepsilon}_{j, c} \leq t\right]-\mathbf{1}\left[e_{j} \leq t\right]-f(t)\left\{e_{j}+\frac{t}{2}\left(e_{j}^{2}-1\right)\right\}\right]\right|=o_{p}\left(n^{-1 / 2}\right),
$$

and we find, uniformly in $t \in \mathbb{R}$,

$$
\hat{\mathbb{F}}_{c}(t)=\frac{1}{n} \sum_{j=1}^{n} \frac{\delta_{j}}{E \delta} \mathbf{1}\left[\hat{\varepsilon}_{j, c} \leq t\right]+o_{p}\left(n^{-1 / 2}\right)=F(t)+\frac{1}{n} \sum_{j=1}^{n} \phi\left(\delta_{j}, e_{j}, t\right)+o_{p}\left(n^{-1 / 2}\right),
$$

where the function $\phi(\delta, e, t)=(\delta / E \delta)\left[\mathbf{1}[e \leq t]-F(t)+f(t)\left\{e+t / 2\left(e^{2}-1\right)\right\}\right]$ is the influence function for $\hat{\mathbb{F}}_{c}$. Since the assumptions of Corollary 3 in Section 2 below are satisfied, it follows for the influence function $\phi$ to be the efficient influence function for estimating $F$, which concludes the proof.

We note the uniform expansion above implies the existence of a functional central limit theorem. In addition, the property that $\hat{\mathbb{F}}_{c}$ is efficient means that competing estimators will not achieve higher precision for large samples. This includes estimators that employ imputation approaches to estimate the missing responses. A consequence of this conclusion is that imputation procedures employed to estimate $F$ may only be effective in small samples. Therefore, we recommend the use of the complete case estimator $\hat{\mathbb{F}}_{c}$ for conducting various hypothesis tests concerning the heteroskedastic MAR model. Section 2 details the remaining results necessary for proving Theorem 2. Section 3 concludes the article with a numerical study of the previous results.

\section{Efficiency}

In this section we will construct the efficient influence function for estimating a linear functional $E[h(e)]$ based on observations of the form $(X, \delta Y, \delta)$, and later specialize this result to $F(t)=E[\mathbf{1}[e \leq t]], t \in \mathbb{R}$. We will first follow the arguments of Chown and Müller (2013), who study this problem for the special case of a constant variance function. In addition, we follow the arguments of Müller, Schick and Wefelmeyer (2006), who consider linear functionals of the joint distribution of $X$ and $Y$ with data of the above form. Finally, we use insight from the 
arguments of Schick (1994), who study estimation of functionals from various heteroskedastic regression models. We only summarize their main arguments and refer the reader to these papers for further details. This allows us to adapt parts of those proofs to the model considered here. Consequently, we only sketch the proofs of the results in this section. To continue, we require Assumption 2 to hold.

In the following, no assumption of a parametric model (finite dimensional) is imposed on any of the regression function, the scale function or the joint distribution of the observations. This means the parameter set $\Theta$ consists of the unknown functions of the statistical model: a family of covariate distributions $\mathscr{G}$ satisfying Assumption 1, a family of error distributions $\mathscr{F}$ that have mean zero, unit variance, finite fourth moment and satisfy Assumption 2, a space of regression functions $\mathscr{R}$ that belong to $H(d, \varphi)$, a space of scale functions $\mathscr{S}$ that is a subspace of $\mathscr{R}$ composed of positive-valued functions and a family of response probability distributions $\mathscr{B}$ that are characterized by the functions from $[0,1]^{m}$ to $(0,1]$. More precisely, $\Theta=\mathscr{G} \times \mathscr{F} \times \mathscr{R} \times \mathscr{S} \times \mathscr{B}$.

We now proceed as in Section 2 of Chown and Müller (2013). Since the construction of the efficient influence function utilizes directional information in $\Theta$, we now identify the set of perturbations $\dot{\Theta}$, which may be thought of as directions. Observe the joint distribution $P(d x, d y, d z)$ takes the form

$$
P(d x, d y, d z)=G(d x) B_{\pi(x)}(d z)\left\{z Q(d y \mid x)+(1-z) \delta_{0}(d y)\right\},
$$

where $B_{p}=p \delta_{1}+(1-p) \delta_{0}$ denotes the Bernoulli distribution with parameter $p$ and $\delta_{t}$ as the Dirac measure at $t$. The model considered here deviates from that considered in Chown and Müller (2013) only in the conditional distribution $Q$ of $Y$ given $X$. This means we first need to consider the spaces $\mathcal{L}_{2,0}(G), \mathcal{L}_{2}\left(G_{\pi}\right)$ and $\mathcal{V}_{0}$. Here $\mathcal{L}_{2,0}(G)$ is the space of functions that are square integrable and have mean zero with respect to $G, \mathcal{L}_{2}\left(G_{\pi}\right)$ is a subspace of $\mathcal{L}_{2}(G)$, where the functions $w$ now satisfy $E\left[w^{2}(X) \pi(X)\{1-\pi(X)\}\right]<\infty$, and $\mathcal{V}_{0}$ is the space of functions satisfying $\int v(x, y) Q(d y \mid x)=0$. It then follows for perturbations $G_{n u}$ of $G, \pi_{n w}$ of $\pi$ and $Q_{n v}$ of $Q$ that are Hellinger differentiable requires the functions $u, w$ and $v$ to be further restricted to appropriate subspaces. Since we have only assumed a model for $Q$, which follows from the heteroskedastic nonparametric regression model (1.2), this only requires resolving the appropriate subspace $\mathcal{V}$ of $\mathcal{V}_{0}$

Using the independence of the covariates $X$ and errors $e$, we may write

$$
\frac{d}{d y} Q(y \mid x)=f\left(\frac{y-r(x)}{\sigma(x)}\right) \frac{1}{\sigma(x)} .
$$

Hence, in order to derive the explicit form of $\mathcal{V}$, we introduce further perturbations $s, t$ and $m$ of the unknown functions $f, r$ and $\sigma$, respectively, and write

$$
\frac{d}{d y} Q_{n v}(y \mid x)=\frac{d}{d y} Q_{n s t m}(y \mid x)=f_{n s}\left(\frac{y-r_{n t}(x)}{\sigma_{n m}(x)}\right) \frac{1}{\sigma_{n m}(x)},
$$

where $f_{n s}(z)=f(z)\left\{1+n^{-1 / 2} s(z)\right\}, r_{n t}(x)=r(x)+n^{-1 / 2} t(x)$ and $\sigma_{n m}(x)=\sigma(x)+n^{-1 / 2} m(x)$ for $s \in \mathcal{S}, t \in \mathcal{L}_{2}\left(G_{1}\right)$ and $m \in \mathcal{L}_{2}\left(G_{1}\right)$. Here

$$
\mathcal{S}=\left\{s \in \mathcal{L}_{2}(F): \int_{-\infty}^{\infty} s(z) f(z) d z=0, \int_{-\infty}^{\infty} z s(z) f(z) d z=0 \text { and } \int_{-\infty}^{\infty} z^{2} s(z) f(z) d z=0\right\},
$$

which is derived by the constraints that $f_{n s}$ must integrate to one, have mean zero and have unit variance. In the following we will write "ㄹ" to denote asymptotic equivalence; i.e. equality up to an additive term of order $o_{p}\left(n^{-1 / 2}\right)$. In addition, we introduce the notation $1(z)=\left(\ell_{1}(z), \ell_{2}(z)\right)^{T}$, for $\ell_{1}(z)=-f^{\prime}(z) / f(z)$ and $\ell_{2}(z)=-1-z f^{\prime}(z) / f(z), \mathbf{k}(x)=(t(x) / \sigma(x), m(x) / \sigma(x))^{T}, \mathbf{e}_{1}=$ $(1,0)^{T}$ and $\mathbf{e}_{2}=(0,1)^{T}$. Similar to the calculations of Chown and Müller (2013) and Schick (1994), who considers, more generally, directionally differentiable regression and scale functions, 
we have, by a brief sketch,

$$
\begin{aligned}
f_{n s} & \left(\frac{y-r_{n t}(x)}{\sigma_{n m}(x)}\right) \frac{1}{\sigma_{n m}(x)} \\
& \doteq f\left(\frac{y-r(x)}{\sigma(x)}\right) \frac{1}{\sigma(x)} \times\left\{1+n^{-1 / 2}\left[\mathbf{k}^{T}(x) 1\left(\frac{y-r(x)}{\sigma(x)}\right)+s\left(\frac{y-r(x)}{\sigma(x)}\right)\right]\right\} .
\end{aligned}
$$

Hence,

$$
\frac{d}{d y} Q_{n s \mathbf{k}}(y \mid x) \doteq f\left(\frac{y-r(x)}{\sigma(x)}\right) \frac{1}{\sigma(x)}\left\{1+n^{-1 / 2}\left[\mathbf{k}^{T}(x) 1\left(\frac{y-r(x)}{\sigma(x)}\right)+s\left(\frac{y-r(x)}{\sigma(x)}\right)\right]\right\}
$$

and $\mathcal{V}$ takes the form

$$
\mathcal{V}=\left\{v(x, y)=\mathbf{k}^{T}(x) 1\left(\frac{y-r(x)}{\sigma(x)}\right)+s\left(\frac{y-r(x)}{\sigma(x)}\right): \mathbf{k} \in \mathcal{L}_{2}\left(G_{1}\right) \times \mathcal{L}_{2}\left(G_{1}\right) \text { and } s \in \mathcal{S}\right\} .
$$

Thus we have perturbations $\dot{\Theta}=\mathcal{L}_{2,0}(G) \times \mathcal{S} \times\left\{\mathcal{L}_{2}\left(G_{1}\right) \times \mathcal{L}_{2}\left(G_{1}\right)\right\} \times \mathcal{L}_{2}\left(G_{\pi}\right)$. Observe, for any $\gamma=(u, s, \mathbf{k}, w)$ in $\dot{\Theta}$, the perturbed distribution $P_{n \gamma}(d x, d y, d z)$ of an observation $(X, \delta Y, \delta)$ is then

$$
P_{n \gamma}(d x, d y, d z)=G_{n u}(d x) B_{\pi_{n w}(x)}(d z)\left\{z Q_{n s \mathbf{k}}(d y \mid x)+(1-z) \delta_{0}(d y)\right\} .
$$

It follows that $P$ is Hellinger differentiable with tangent

$$
d_{\gamma}(X, \delta Y, \delta)=u(X)+\{\delta-\pi(X)\} w(X)+\delta\left\{\mathbf{k}^{T}(X) 1(e)+s(e)\right\}
$$

and we arrive at the form of the tangent space as

$$
T=\mathcal{L}_{2,0}(G) \oplus\left\{\{\delta-\pi(X)\} w(X): w \in \mathcal{L}_{2}\left(G_{\pi}\right)\right\} \oplus\{\delta v(X, Y): v \in \mathcal{V}\} .
$$

Consequently, we have local asymptotic normality. This means the following expansion holds:

$$
\sum_{j=1}^{n} \log \left(\frac{d P_{n \gamma}}{d P}\left(X_{j}, \delta_{j} Y_{j}, \delta_{j}\right)\right)=n^{-1 / 2} \sum_{j=1}^{n} d_{\gamma}\left(X_{j}, \delta_{j} Y_{j}, \delta_{j}\right)-\frac{1}{2} E\left[d_{\gamma}^{2}(X, \delta Y, \delta)\right]+o_{p}(1),
$$

where $d P$ denotes the density function of $P$.

We are interested in the linear functional $E[h(e)]$. In order to specify a gradient for $E[h(e)]$, we first need to find its directional derivative $\gamma_{h} \in \dot{\Theta}$, which is characterized by a limit as follows. As in Müller, Schick and Wefelmeyer (2004), we have, for every $s \in S$,

$$
\lim _{n \rightarrow \infty} n^{1 / 2}\left[\int_{-\infty}^{\infty} h(z) f_{n s}(z) d z-\int_{-\infty}^{\infty} h(z) f(z) d z\right]=E[h(e) s(e)]=E\left[h_{0}(e) s(e)\right],
$$

with $h_{0}$ given as a projection of $h$ onto $\mathcal{S}$ :

$$
\begin{aligned}
h_{0}(z)= & h(z)-E[h(e)]-z E[e h(e)] \\
& -\frac{z^{2}-E\left[e^{3}\right] z-1}{E\left[e^{4}\right]-E^{2}\left[e^{3}\right]-1}\left\{E\left[e^{2} h(e)\right]-E\left[e^{3}\right] E[e h(e)]-E[h(e)]\right\} .
\end{aligned}
$$

Thus, $E[h(e)]$ is directionally differentiable with directional derivative $\left(0, h_{0}, \mathbf{0}, 0\right)$ and gradient $h_{0}(e)$. By the convolution theorem (see, for example, Section 2 of Schick, 1993) the unique canonical gradient $g^{*}(X, \delta Y, \delta)$ is found by orthogonally projecting the gradient $h_{0}(e)$ onto the tangent space $T$. Thus, $g^{*}(X, \delta Y, \delta)$ must take the form

$$
g^{*}(X, \delta Y, \delta)=u^{*}(X)+\{\delta-\pi(X)\} w^{*}(X)+\delta\left\{\mathbf{k}^{* T}(X) 1(e)+s^{*}(e)\right\} .
$$

Now proceeding as in Section 2 of Chown and Müller (2013), we obtain the following result:

LEMma 1. The canonical gradient of $E[h(e)]$ is $g^{*}(X, \delta Y, \delta)$, which is characterized by $\left(0, s^{*}, \mathbf{k}^{*}, 0\right)$, where

$$
s^{*}(z)=\frac{1}{E \delta} h_{0}(z)-E_{1}\left[\mathbf{k}^{* T}(X)\right] 1_{0}(z) \quad \text { and } \quad \mathbf{k}^{*} \equiv-\frac{1}{E \delta} J_{d}^{-1} E\left[1_{0}(e) h_{0}(e)\right]
$$


with $h_{0}$ given above and the quantities

$$
1_{0}(z)=1(z)-z \mathbf{e}_{1}-\frac{z^{2}-E\left[e^{3}\right] z-1}{E\left[e^{4}\right]-E^{2}\left[e^{3}\right]-1}\left\{2 \mathbf{e}_{2}-E\left[e^{3}\right] \mathbf{e}_{1}\right\}
$$

and

$$
J_{d}^{-1}=\frac{1}{E\left[e^{4}\right]-E^{2}\left[e^{3}\right]-1}\left[\begin{array}{cc}
E\left[e^{4}\right]-1 & -2 E\left[e^{3}\right] \\
-2 E\left[e^{3}\right] & 4
\end{array}\right] .
$$

We will call an estimator $\hat{\mu}$ for $E[h(e)]$ efficient, in the sense of Hájek and Le Cam, if it is asymptotically linear with corresponding influence function equal to the canonical gradient $g^{*}(X, \delta Y, \delta)$ that characterizes $E[h(e)]$. This means $\hat{\mu}$ satisfies the expansion

$$
n^{1 / 2}\{\hat{\mu}-E[h(e)]\}=n^{-1 / 2} \sum_{j=1}^{n} g^{*}\left(X_{j}, \delta_{j} Y_{j}, \delta_{j}\right)+o_{p}(1) .
$$

We combine this fact with Lemma 1 and (2.1) to obtain the following result:

THEOREM 3. Consider the heteroskedastic nonparametric regression model with responses missing at random. An estimator $\hat{\mu}$ of $E[h(e)]$ is efficient, if it satisfies the expansion

$$
n^{1 / 2}\{\hat{\mu}-E[h(e)]\}=n^{-1 / 2} \sum_{j=1}^{n} \frac{\delta}{E \delta}\left[h_{0}\left(e_{j}\right)-E^{T}\left[h_{0}\left(e_{j}\right) l_{0}\left(e_{j}\right)\right] J_{d}^{-1} 1_{d}\left(e_{j}\right)\right]+o_{p}(1),
$$

where $h_{0}$ is given above, $1_{0}$ and $J_{d}^{-1}$ are given in Lemma 1 and

$$
1_{d}(z)=z \mathbf{e}_{1}+\frac{z^{2}-z E\left[e^{3}\right]-1}{E\left[e^{4}\right]-E^{2}\left[e^{3}\right]-1}\left\{2 \mathbf{e}_{2}-E\left[e^{3}\right] \mathbf{e}_{1}\right\}
$$

In this article, we are interested in the function $h(z)=\mathbf{1}[z \leq t]$ because we estimate $F(t)=E[\mathbf{1}[e \leq t]]$ using $\hat{\mathbb{F}}_{c}$. We now obtain, using Theorem 3 with this $h$, the expansion for an efficient estimator of the error distribution function $F$.

COROllary 3. Consider the heteroskedastic nonparametric regression model with responses missing at random. An estimator $\hat{F}$ of $F$ is efficient, in the sense of Hájek and Le Cam, if it satisfies the expansion

$$
n^{1 / 2}\{\hat{F}(t)-F(t)\}=n^{-1 / 2} \sum_{j=1}^{n} \frac{\delta}{E \delta}\left[\mathbf{1}\left[e_{j} \leq t\right]-F(t)+f(t)\left\{e_{j}+\frac{t}{2}\left(e_{j}^{2}-1\right)\right\}\right]+o_{p}(1) .
$$

\section{Simulations}

We conclude this article with a small numerical study of the previous results. In the following we work with

$$
r\left(x_{1}, x_{2}\right)=1+x_{1}-x_{2}+2 e^{-\frac{1}{2} \sqrt{x_{1}^{2}+x_{2}^{2}}} \text { and } \sigma\left(x_{1}, x_{2}\right)=\sqrt{1+2 x_{1}^{2}+2 x_{2}^{2}}
$$

to preserve the nonparametric nature of the study. The covariates $X_{1}$ and $X_{2}$ are each randomly generated from a $U(-1,1)$ distribution, and the errors $e$ are generated from a standard normal distribution. The indicators $\delta$ are randomly generated from a $\operatorname{Bernoulli}\left(\pi\left(X_{1}, X_{2}\right)\right)$ distribution, with $\pi\left(X_{1}, X_{2}\right)=P\left(\delta=1 \mid X_{1}, X_{2}\right)$. Here we use $\pi\left(x_{1}, x_{2}\right)=1-1 /\left(1+e^{-\left(x_{1}+x_{2}\right) / 2}\right)$. Consequently, the average amount of missing data is about $50 \%$ (ranging between $26 \%$ and $74 \%$ ). We work with $d=3$, the locally cubic smoother, to estimate both of the functions $r$ and $\sigma$. For our choice of using a product of tricubic kernel functions and bandwidth $\lambda_{n}=3(n \log (n))^{-1 / 7}$, the assumptions of Theorem 2 are satisfied.

To check the performance of our proposed estimator, we have conducted simulations of 1000 runs using samples of sizes 100, 200, 500 and 1000. The distribution function has been estimated at the points $0,-1,-2$ and -3 (the results for t-values 1,2 , and 3 are very similar). 


\begin{tabular}{|r|cccc|}
\hline$n$ & -3 & -2 & -1 & 0 \\
\hline 100 & $0.0977(0.0231)$ & $0.0563(0.0588)$ & $0.0030(0.1516)$ & $-0.0318(0.1957)$ \\
200 & $0.0965(0.0241)$ & $0.0777(0.0722)$ & $0.1646(0.1555)$ & $-0.0818(0.2124)$ \\
500 & $0.0301(0.0089)$ & $0.0008(0.0496)$ & $0.1806(0.1285)$ & $-0.0746(0.2022)$ \\
1000 & $0.0006(0.0030)$ & $-0.0382(0.0348)$ & $0.1389(0.1033)$ & $-0.0826(0.1848)$ \\
\hline
\end{tabular}

TABLE 1. Simulated asymptotic bias and variance (in parentheses), at the points $-3,-2,-1$ and 0 , of $n^{-1 / 2}\left\{\hat{\mathbb{F}}_{c}-F\right\}$.

\begin{tabular}{|r|cccc|c|}
\hline$n$ & -3 & -2 & -1 & 0 & AMISE \\
\hline 100 & 0.0326 & 0.0619 & 0.1516 & 0.1967 & 0.8248 \\
200 & 0.0334 & 0.0782 & 0.1826 & 0.2191 & 0.9248 \\
500 & 0.0098 & 0.0496 & 0.1611 & 0.2077 & 0.7184 \\
1000 & 0.0030 & 0.0362 & 0.1226 & 0.1916 & 0.5812 \\
$\infty$ & 0.0025 & 0.0270 & 0.0913 & 0.1817 & 0.4231 \\
\hline
\end{tabular}

TABLE 2. Simulated asymptotic mean squared error, at the points $-3,-2,-1$ and 0 , and asymptotic mean integrated squared error of $n^{-1 / 2}\left\{\hat{\mathbb{F}}_{c}-F\right\}$.

Table 1 shows the results of the simulated asymptotic bias and variance of $\hat{\mathbb{F}}_{c}$, which is calculated by multiplying the simulated bias by the square-root of each sample size and multiplying the simulated variance by each sample size. These quantities are predicted to be stable across sample sizes by Theorem 2, and, therefore, will change only with the value of $t$. Table 2 shows the results of the simulated asymptotic mean squared error (AMSE) and the simulated asymptotic mean integrated squared error (AMISE), which are calculated similarly to the simulated asymptotic variance. In addition, we have calculated the AMSE and AMISE for an infinitely large sample using the results of Theorem 2, which are given by the figures labeled with sample size $\infty$.

Beginning with Table 1, we can see the asymptotic bias in $\hat{\mathbb{F}}_{c}$ is slightly negative near zero, increases to become positive when moving away from zero and, finally, decreases toward zero again when moving into the tails of the distribution. This is in contrast to the asymptotic variance, which appears to be largest near zero and only decreases toward zero when moving into the tails of the distribution. Nevertheless, we can see the values appear reasonably stable at the larger sample sizes 500 and 1000 as desired. Turning our attention now to Table 2, we can plainly see the estimator $\hat{\mathbb{F}}_{c}$ appears to have both AMSE and AMISE values decreasing toward the respective predicted limiting values (given by the $\infty$ figures). This indicates the predictions made by Theorem 2 are indeed adequate for describing the limiting behavior of $\hat{\mathbb{F}}_{c}$. In conclusion we find the complete case estimator $\hat{\mathbb{F}}_{c}$ useful and practical for estimating the distribution of the errors $F$ in the heteroskedastic MAR model.

\section{Appendix}

This section is the proof of Theorem 1, which is, in particular, concerned with data obtained from a full model.

Proof of Theorem 1. To begin, we decompose the stochastic quantity in the absolute brackets in the left-hand side of the assertion into a sum of three remainder terms:

$R_{1}(t)=\frac{1}{n} \sum_{j=1}^{n}\left\{\mathbf{1}\left[\hat{\varepsilon}_{j} \leq t\right]-E\left[F\left(t+\frac{\hat{r}(X)-r(X)}{\sigma(X)}+t \frac{\hat{\sigma}(X)-\sigma(X)}{\sigma(X)}\right) \mid \mathbb{D}\right]-\mathbf{1}\left[e_{j} \leq t\right]+F(t)\right\}$, 


$$
\begin{aligned}
R_{2}(t)= & E\left[F\left(t+\frac{\hat{r}(X)-r(X)}{\sigma(X)}+t \frac{\hat{\sigma}(X)-\sigma(X)}{\sigma(X)}\right) \mid \mathbb{D}\right]-F(t) \\
& -f(t) \int_{[0,1]^{m}} \frac{\hat{r}(x)-r(x)}{\sigma(x)} g(x) d x-t f(t) \int_{[0,1]^{m}} \frac{\hat{\sigma}(x)-\sigma(x)}{\sigma(x)} g(x) d x
\end{aligned}
$$

and

$$
\begin{aligned}
R_{3}(t)= & f(t)\left(\int_{[0,1]^{m}} \frac{\hat{r}(x)-r(x)}{\sigma(x)} g(x) d x-\frac{1}{n} \sum_{j=1}^{n} e_{j}\right) \\
& +t f(t)\left(\int_{[0,1]^{m}} \frac{\hat{\sigma}(x)-\sigma(x)}{\sigma(x)} g(x) d x-\frac{1}{n} \sum_{j=1}^{n} \frac{e_{j}^{2}-1}{2}\right) .
\end{aligned}
$$

The proof will be concluded once we have shown $\sup _{t \in \mathbb{R}}\left|R_{i}(t)\right|=o_{p}\left(n^{-1 / 2}\right)$ for each $i=1,2,3$.

To show $\sup _{t \in \mathbb{R}}\left|R_{1}(t)\right|=o_{p}\left(n^{-1 / 2}\right)$, we will proceed similarly as in the proof of Theorem 2.1 of Neumeyer and Van Keilegom (2010), and we refer the reader to that paper for further details. The main difference between the proof techniques lies in the details concerning the estimators $\hat{r}$ and $\hat{\sigma}$, which require us to use an approximation argument the previous authors can avoid. We begin this argument by noting analogous conclusions of Corollary 1 hold for the local polynomial estimators $\hat{r}$ and $\hat{r}_{2}$, which follows from the discussion in Remark 1 . This means there are random functions $\hat{a}_{1}$ (associated with $\hat{r}$ ) and $\hat{a}_{2}$ (associated with $\hat{r}_{2}$ ) that satisfy $P\left(\hat{a}_{1} \in H_{1}(m, \alpha)\right) \rightarrow 1$ and $P\left(\hat{a}_{2} \in H_{1}(m, \alpha)\right) \rightarrow 1$, as $n \rightarrow \infty$, for some $\alpha>0$, $\sup _{x \in[0,1]^{m}}\left|\hat{r}(x)-r(x)-\hat{a}_{1}(x)\right|=o_{p}\left(n^{-1 / 2}\right)$ and $\sup _{x \in[0,1]^{m}}\left|\hat{r}_{2}(x)-r_{2}(x)-\hat{a}_{2}(x)\right|=o_{p}\left(n^{-1 / 2}\right)$. In their Lemma A.3, Neumeyer and Van Keilegom (2010) show a class of functions similar to

$$
\begin{aligned}
\mathfrak{F}=\{(x, z) \mapsto & \mathbf{1}\left[z \leq t+\left\{1-t \frac{r(x)}{\sigma(x)}\right\} \frac{a_{1}(x)}{\sigma(x)}+\frac{t}{2} \frac{a_{2}(x)}{\sigma^{2}(x)}\right] \\
& \left.-E\left[F\left(t+\left\{1-t \frac{r(X)}{\sigma(X)}\right\} \frac{a_{1}(X)}{\sigma(X)}+\frac{t}{2} \frac{a_{2}(X)}{\sigma^{2}(X)}\right)\right]: t \in \mathbb{R}, a_{1}, a_{2} \in H_{1}(m, \alpha)\right\}
\end{aligned}
$$

is $G \times F$-Donsker, and, since our argument that $\mathfrak{F}$ is also $G \times F$-Donsker is very similar, it is omitted. It then follows by Corollary 2.3.12 in van der Vaart and Wellner (1996) for the stochastic equicontinuity condition for empirical processes ranging over $\mathfrak{F}$ to hold, i.e. writing $\mathfrak{f}$ for the form of the map in the definition of $\mathfrak{F}$ above, we have, for any $\epsilon>0$,

$$
\lim _{\alpha \downarrow 0} \limsup _{n \rightarrow \infty} P\left(\sup _{\mathfrak{f}_{1}, \mathfrak{f}_{2} \in \mathfrak{F}: \operatorname{Var}\left[\mathfrak{f}_{1}(X, e)-\mathfrak{f}_{2}(X, e)\right] \leq \alpha} n^{-1 / 2}\left|\sum_{j=1}^{n}\left\{\mathfrak{f}_{1}\left(X_{j}, e_{j}\right)-\mathfrak{f}_{2}\left(X_{j}, e_{j}\right)\right\}\right|>\epsilon\right)=0 .
$$

We will now use equicontinuity of empirical processes indexed by $\mathfrak{F}$ to finish proving the assertion. In what follows we may assume that $\hat{a}_{1}$ and $\hat{a}_{2}$ belong to $H_{1}(m, \alpha)$, which we have already shown is an event with probability tending to one as $n$ increases. Hence, we have $\mathfrak{f}_{t, \hat{a}_{1}, \hat{a}_{2} \in} \in$ $\mathfrak{F}$, where now the expected value is conditional on the data $\mathbb{D}=\left\{\left(X_{1}, Y_{1}\right), \ldots,\left(X_{n}, Y_{n}\right)\right\}$ and we have chosen $\hat{a}_{1}$ for $a_{1}$ and $\hat{a}_{2}$ for $a_{2}$ in the definition of $\mathfrak{F}$. We also have $\mathfrak{f}_{t, 0,0} \in \mathfrak{F}$, which now corresponds with choosing the zero function for both of $a_{1}$ and $a_{2}$ in the definition of $\mathfrak{F}$ above. Inspecting page 961 of the proof of Lemma 1 of Müller, Schick and Wefelmeyer (2009) shows for their situation $\sup _{x \in[0,1]^{m}}|\hat{a}(x)|=o_{p}(1)$, which continues to hold in the present situation, i.e. both $\hat{a}_{1}$ and $\hat{a}_{2}$ satisfy

$$
\sup _{x \in[0,1]^{m}}\left|\hat{a}_{1}(x)\right|=o_{p}(1) \quad \text { and } \sup _{x \in[0,1]^{m}}\left|\hat{a}_{2}(x)\right|=o_{p}(1) .
$$

In order to apply (4.1), we need to consider the variation of the difference $\mathfrak{f}_{t, \hat{a}_{1}, \hat{a}_{2}}(X, e)-$ $\mathfrak{f}_{t, 0,0}(X, e)$ and find that it is asymptotically negligible. To check the variance condition beneath the supremum in (4.1) is satisfied, we calculate $\operatorname{Var}\left[\mathfrak{f}_{t, \hat{a}_{1}, \hat{a}_{2}}(X, e)-\mathfrak{f}_{t, 0,0}(X, e) \mid \mathbb{D}\right]$ and find it is 
equal to

$$
\begin{aligned}
& E\left[\left\{1\left[e \leq t+\left\{1-t \frac{r(X)}{\sigma(X)}\right\} \frac{\hat{a}_{1}(X)}{\sigma(X)}+\frac{t}{2} \frac{\hat{a}_{2}(X)}{\sigma^{2}(X)}\right]\right.\right. \\
& \left.\left.-E\left[F\left(t+\left\{1-t \frac{r(X)}{\sigma(X)}\right\} \frac{\hat{a}_{1}(X)}{\sigma(X)}+\frac{t}{2} \frac{\hat{a}_{2}(X)}{\sigma^{2}(X)}\right) \mid \mathbb{D}\right]\right\}^{2} \mid \mathbb{D}\right] \\
& -2 E\left[\left\{1\left[e \leq t+\left\{1-t \frac{r(X)}{\sigma(X)}\right\} \frac{\hat{a}_{1}(X)}{\sigma(X)}+\frac{t}{2} \frac{\hat{a}_{2}(X)}{\sigma^{2}(X)}\right]\right.\right. \\
& \left.\left.-E\left[F\left(t+\left\{1-t \frac{r(X)}{\sigma(X)}\right\} \frac{\hat{a}_{1}(X)}{\sigma(X)}+\frac{t}{2} \frac{\hat{a}_{2}(X)}{\sigma^{2}(X)}\right) \mid \mathbb{D}\right]\right\} \times\{\mathbf{1}[e \leq t]-F(t)\} \mid \mathbb{D}\right] \\
& +E\left[\{\mathbf{1}[e \leq t]-F(t)\}^{2}\right] \\
& =E\left[F\left(t+\left\{1-t \frac{r(X)}{\sigma(X)}\right\} \frac{\hat{a}_{1}(X)}{\sigma(X)}+\frac{t}{2} \frac{\hat{a}_{2}(X)}{\sigma^{2}(X)}\right) \mid \mathbb{D}\right] \\
& -E^{2}\left[F\left(t+\left\{1-t \frac{r(X)}{\sigma(X)}\right\} \frac{\hat{a}_{1}(X)}{\sigma(X)}+\frac{t}{2} \frac{\hat{a}_{2}(X)}{\sigma^{2}(X)}\right) \mid \mathbb{D}\right] \\
& +F(t)-F^{2}(t)-2 E\left[F\left(\min \left\{t, t+\left\{1-t \frac{r(X)}{\sigma(X)}\right\} \frac{\hat{a}_{1}(X)}{\sigma(X)}+\frac{t}{2} \frac{\hat{a}_{2}(X)}{\sigma^{2}(X)}\right\}\right) \mid \mathbb{D}\right] \\
& +2 F(t) E\left[F\left(t+\left\{1-t \frac{r(X)}{\sigma(X)}\right\} \frac{\hat{a}_{1}(X)}{\sigma(X)}+\frac{t}{2} \frac{\hat{a}_{2}(X)}{\sigma^{2}(X)}\right) \mid \mathbb{D}\right] \\
& =E\left[F\left(t+\left\{1-t \frac{r(X)}{\sigma(X)}\right\} \frac{\hat{a}_{1}(X)}{\sigma(X)}+\frac{t}{2} \frac{\hat{a}_{2}(X)}{\sigma^{2}(X)}\right)\right. \\
& \left.-F\left(\min \left\{t, t+\left\{1-t \frac{r(X)}{\sigma(X)}\right\} \frac{\hat{a}_{1}(X)}{\sigma(X)}+\frac{t}{2} \frac{\hat{a}_{2}(X)}{\sigma^{2}(X)}\right\}\right) \mid \mathbb{D}\right] \\
& +E\left[F(t)-F\left(\min \left\{t, t+\left\{1-t \frac{r(X)}{\sigma(X)}\right\} \frac{\hat{a}_{1}(X)}{\sigma(X)}+\frac{t}{2} \frac{\hat{a}_{2}(X)}{\sigma^{2}(X)}\right\}\right) \mid \mathbb{D}\right] \\
& +\left\{E\left[F(t)-F\left(t+\left\{1-t \frac{r(X)}{\sigma(X)}\right\} \frac{\hat{a}_{1}(X)}{\sigma(X)}+\frac{t}{2} \frac{\hat{a}_{2}(X)}{\sigma^{2}(X)}\right) \mid \mathbb{D}\right]\right\} \\
& \times E\left[F\left(t+\left\{1-t \frac{r(X)}{\sigma(X)}\right\} \frac{\hat{a}_{1}(X)}{\sigma(X)}+\frac{t}{2} \frac{\hat{a}_{2}(X)}{\sigma^{2}(X)}\right) \mid \mathbb{D}\right] \\
& +\left\{E\left[F\left(t+\left\{1-t \frac{r(X)}{\sigma(X)}\right\} \frac{\hat{a}_{1}(X)}{\sigma(X)}+\frac{t}{2} \frac{\hat{a}_{2}(X)}{\sigma^{2}(X)}\right)-F(t) \mid \mathbb{D}\right]\right\} \times F(t) \\
& =E\left[F\left(\max \left\{t, t+\left\{1-t \frac{r(X)}{\sigma(X)}\right\} \frac{\hat{a}_{1}(X)}{\sigma(X)}+\frac{t}{2} \frac{\hat{a}_{2}(X)}{\sigma^{2}(X)}\right\}\right)\right. \\
& \left.-F\left(\min \left\{t, t+\left\{1-t \frac{r(X)}{\sigma(X)}\right\} \frac{\hat{a}_{1}(X)}{\sigma(X)}+\frac{t}{2} \frac{\hat{a}_{2}(X)}{\sigma^{2}(X)}\right\}\right) \mid \mathbb{D}\right] \\
& -E^{2}\left[F\left(\max \left\{t, t+\left\{1-t \frac{r(X)}{\sigma(X)}\right\} \frac{\hat{a}_{1}(X)}{\sigma(X)}+\frac{t}{2} \frac{\hat{a}_{2}(X)}{\sigma^{2}(X)}\right\}\right)\right. \\
& \left.-F\left(\min \left\{t, t+\left\{1-t \frac{r(X)}{\sigma(X)}\right\} \frac{\hat{a}_{1}(X)}{\sigma(X)}+\frac{t}{2} \frac{\hat{a}_{2}(X)}{\sigma^{2}(X)}\right\}\right) \mid \mathbb{D}\right] \text {. }
\end{aligned}
$$

Therefore, we find

$$
\begin{aligned}
& \sup _{t \in \mathbb{R}} \operatorname{Var}\left[\mathfrak{f}_{t, \hat{a}_{1}, \hat{a}_{2}}(X, e)-\mathfrak{f}_{t, 0,0}(X, e) \mid \mathbb{D}\right] \\
& \leq E\left[F\left(\max \left\{t, t+\left\{1-t \frac{r(X)}{\sigma(X)}\right\} \frac{\hat{a}_{1}(X)}{\sigma(X)}+\frac{t}{2} \frac{\hat{a}_{2}(X)}{\sigma^{2}(X)}\right\}\right)\right.
\end{aligned}
$$




$$
\begin{aligned}
& \left.-F\left(\min \left\{t, t+\left\{1-t \frac{r(X)}{\sigma(X)}\right\} \frac{\hat{a}_{1}(X)}{\sigma(X)}+\frac{t}{2} \frac{\hat{a}_{2}(X)}{\sigma^{2}(X)}\right\}\right) \mid \mathbb{D}\right] \\
\leq & \left(\sup _{t \in \mathbb{R}} f(t)\left[\inf _{x \in[0,1]^{m}} \sigma(x)\right]^{-1}+\sup _{t \in \mathbb{R}}|t f(t)| \sup _{x \in[0,1]^{m}}|r(x)|\left[\inf _{x \in[0,1]^{m}} \sigma(x)\right]^{-2}\right) \sup _{x \in[0,1]^{m}}\left|\hat{a}_{1}(x)\right| \\
& +\frac{1}{2} \sup _{t \in \mathbb{R}}|t f(t)|\left[\inf _{x \in[0,1]^{m}} \sigma(x)\right]^{-2} \sup _{x \in[0,1]^{m}}\left|\hat{a}_{2}(x)\right| .
\end{aligned}
$$

It then follows that $\operatorname{Var}\left[\mathfrak{f}_{t, \hat{a}_{1}, \hat{a}_{2}}(X, e)-\mathfrak{f}_{t, 0,0}(X, e) \mid \mathbb{D}\right]=o_{p}(1), t \in \mathbb{R}$, from the results above, i.e. the variance is asymptotically negligible. Hence, have asymptotic equicontinuity for empirical processes indexed by the elements of $\mathfrak{F}$ corresponding to the choices $\hat{a}_{1}$ and $\hat{a}_{2}$, for $a_{1}$ and $a_{2}$, and the zero function, in place of both $a_{1}$ and $a_{2}$. This implies

$$
\sup _{t \in \mathbb{R}}\left|\frac{1}{n} \sum_{j=1}^{n}\left\{\mathfrak{f}_{t, \hat{a}_{1}, \hat{a}_{2}}\left(X_{j}, e_{j}\right)-\mathfrak{f}_{t, 0,0}\left(X_{j}, e_{j}\right)\right\}\right|=o_{p}\left(n^{-1 / 2}\right) .
$$

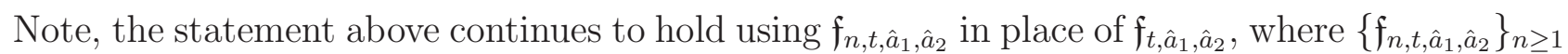
is any sequence of functions from $\mathfrak{F}$ converging to the same limit as $\mathfrak{f}_{t, \hat{a}_{1}, \hat{a}_{2}}$ (i.e. $\left.\mathfrak{f}_{t, 0,0}\right)$, as $n$ increases, that continues to satisfy the variation condition above, which will be an important observation for the arguments that follow.

We can bound $\sup _{t \in \mathbb{R}}\left|R_{1}(t)\right|$ by the sum of the left-hand side of (4.2) and

$$
\sup _{t \in \mathbb{R}}\left|\frac{1}{n} \sum_{j=1}^{n}\left\{\chi_{t}\left(X_{j}, e_{j}\right)-\mathfrak{f}_{t, \hat{a}_{1}, \hat{a}_{2}}\left(X_{j}, e_{j}\right)\right\}\right|
$$

where $\chi_{t}\left(X_{j}, e_{j}\right), j=1, \ldots, n$ and $t \in \mathbb{R}$, is equal to

$$
\mathbf{1}\left[e_{j} \leq t+\frac{\hat{r}\left(X_{j}\right)-r\left(X_{j}\right)}{\sigma\left(X_{j}\right)}+t \frac{\hat{\sigma}\left(X_{j}\right)-\sigma\left(X_{j}\right)}{\sigma\left(X_{j}\right)}\right]-E\left[F\left(t+\frac{\hat{r}(X)-r(X)}{\sigma(X)}+t \frac{\hat{\sigma}(X)-\sigma(X)}{\sigma(X)}\right) \mid \mathbb{D}\right] .
$$

To continue, we will require some auxiliary results. By a direct application of Theorem 6 of Masry (1996), we have $\sup _{x \in[0,1]^{m}}\{\hat{r}(x)-r(x)\}^{2}=o\left(n^{-1 / 2}\right), \sup _{x \in[0,1]^{m}}\left\{\hat{r}_{2}(x)-r_{2}(x)\right\}^{2}=$ $o\left(n^{-1 / 2}\right)$ and $\sup _{x \in[0,1]^{m}}|\hat{r}(x)-r(x)|\left|\hat{r}_{2}(x)-r_{2}(x)\right|=o\left(n^{-1 / 2}\right)$, almost surely. Now write

$$
\hat{\sigma}(x)-\sigma(x)=\frac{\hat{\sigma}^{2}(x)-\sigma^{2}(x)}{2 \sigma(x)}-\frac{\{\hat{\sigma}(x)-\sigma(x)\}^{2}}{2 \sigma(x)}
$$

and

$$
\hat{r}^{2}(x)-r^{2}(x)=2 r(x)\{\hat{r}(x)-r(x)\}+\{\hat{r}(x)-r(x)\}^{2} .
$$

Together these results imply

$$
\hat{\sigma}(x)-\sigma(x)=\frac{\hat{r}_{2}(x)-r_{2}(x)}{2 \sigma(x)}-\frac{r(x)}{\sigma(x)}\{\hat{r}(x)-r(x)\}-\frac{\{\hat{r}(x)-r(x)\}^{2}}{2 \sigma(x)}-\frac{\{\hat{\sigma}(x)-\sigma(x)\}^{2}}{2 \sigma(x)}
$$

and

$$
\begin{aligned}
\{\hat{\sigma}(x)-\sigma(x)\}^{2}= & \frac{\left\{\hat{r}_{2}(x)-r(x)\right\}^{2}}{4 \sigma^{2}(x)}-\frac{r(x)}{\sigma^{2}(x)}\left\{\hat{r}_{2}(x)-r_{2}(x)\right\}\{\hat{r}(x)-r(x)\} \\
& -\frac{\left\{\hat{r}_{2}(x)-r_{2}(x)\right\}\{\hat{r}(x)-r(x)\}^{2}}{2 \sigma^{2}(x)}-\frac{\left\{\hat{r}_{2}(x)-r_{2}(x)\right\}\{\hat{\sigma}(x)-\sigma(x)\}^{2}}{2 \sigma^{2}(x)} \\
& +\frac{r^{2}(x)}{\sigma^{2}(x)}\{\hat{r}(x)-r(x)\}^{2}+\frac{r(x)}{\sigma^{2}(x)}\{\hat{r}(x)-r(x)\}^{3} \\
& +\frac{r(x)}{\sigma^{2}(x)}\{\hat{r}(x)-r(x)\}\{\hat{\sigma}(x)-\sigma(x)\}^{2}+\frac{\{\hat{r}(x)-r(x)\}^{4}}{4 \sigma^{2}(x)} \\
& +\frac{\{\hat{r}(x)-r(x)\}^{2}\{\hat{\sigma}(x)-\sigma(x)\}^{2}}{2 \sigma^{2}(x)}+\frac{\{\hat{\sigma}(x)-\sigma(x)\}^{4}}{4 \sigma^{2}(x)} .
\end{aligned}
$$


Combining the last statement with the results above, we find $\sup _{x \in[0,1]^{m}}\{\hat{\sigma}(x)-\sigma(x)\}^{2}=$ $o\left(n^{-1 / 2}\right)$, almost surely. Using the definitions of $\hat{r}$ and $\hat{\sigma}$, we find $t+\{\hat{r}(x)-r(x)\} / \sigma(x)+$ $t\{\hat{\sigma}(x)-\sigma(x)\} / \sigma(x)$ is equal to

$$
\begin{gathered}
t\left\{1+\frac{\hat{r}_{2}(x)-r_{2}(x)-\hat{a}_{2}(x)}{2 \sigma^{2}(x)}-\frac{r(x)}{\sigma(x)} \frac{\hat{r}(x)-r(x)-\hat{a}_{1}(x)}{\sigma(x)}-\frac{\{\hat{r}(x)-r(x)\}^{2}}{2 \sigma^{2}(x)}-\frac{\{\hat{\sigma}(x)-\sigma(x)\}^{2}}{2 \sigma^{2}(x)}\right\} \\
+\frac{\hat{r}(x)-r(x)-\hat{a}_{1}(x)}{\sigma(x)}+\left\{1-t \frac{r(x)}{\sigma(x)}\right\} \frac{\hat{a}_{1}(x)}{\sigma(x)}+\frac{t}{2} \frac{\hat{a}_{2}(x)}{\sigma^{2}(x)} .
\end{gathered}
$$

This means we can appropriately choose random sequences $\left\{u_{n}\right\}_{n \geq 1}$ and $\left\{v_{n}\right\}_{n \geq 1}$, where

$$
\begin{aligned}
u_{n}=4\left[\inf _{x \in[0,1]^{m}} \sigma(x)\right]^{-2} \max \left\{\frac{n^{1 / 2}}{2} \sup _{x \in[0,1]^{m}}\left|\hat{r}_{2}(x)-r(x)-\hat{a}_{2}(x)\right|,\right. \\
\\
n^{1 / 2} \sup _{x \in[0,1]^{m}}|r(x)| \sup _{x \in[0,1]^{m}}\left|\hat{r}(x)-r(x)-\hat{a}_{1}(x)\right|, \\
\left.\quad \frac{n^{1 / 2}}{2} \sup _{x \in[0,1]^{m}}\{\hat{r}(x)-r(x)\}^{2}, \frac{n^{1 / 2}}{2} \sup _{x \in[0,1]^{m}}\{\hat{\sigma}(x)-\sigma(x)\}^{2}\right\} \\
=o_{p}(1)
\end{aligned}
$$

and

$$
v_{n}=n^{1 / 2}\left[\inf _{x \in[0,1]^{m}} \sigma(x)\right]^{-1} \sup _{x \in[0,1]^{m}}\left|\hat{r}(x)-r(x)-\hat{a}_{1}(x)\right|=o_{p}(1),
$$

which depend only on $\mathbb{D}$. We then define function sequences $\mathfrak{f}_{n, t, \hat{a}_{1}, \hat{a}_{2}}^{++}, \mathfrak{f}_{n, t, \hat{a}_{1}, \hat{a}_{2}}^{+-} \mathfrak{f}_{n, t, \hat{a}_{1}, \hat{a}_{2}}^{-+}$and $\mathfrak{f}_{n, t, \hat{a}_{1}, \hat{a}_{2}}^{--}$from $\mathfrak{F}$ as follows. Define the sequences $\mathfrak{f}_{n, t, \hat{a}_{1}, \hat{a}_{2}}^{++}$and $\mathfrak{f}_{n, t, \hat{a}_{1}, \hat{a}_{2}}^{--}$as

$$
\begin{aligned}
\mathfrak{f}_{n, t, \hat{a}_{1}, \hat{a}_{2}}^{++}(x, z)= & \mathbf{1}\left[z \leq t\left\{1+n^{-1 / 2} u_{n}\right\}+n^{-1 / 2} v_{n}+\left\{1-t \frac{r(x)}{\sigma(x)}\right\} \frac{\hat{a}_{1}(x)}{\sigma(x)}+\frac{t}{2} \frac{\hat{a}_{2}(x)}{\sigma^{2}(x)}\right] \\
& -E\left[F\left(t\left\{1+n^{-1 / 2} u_{n}\right\}+n^{-1 / 2} v_{n}+\left\{1-t \frac{r(X)}{\sigma(X)}\right\} \frac{\hat{a}_{1}(X)}{\sigma(X)}+\frac{t}{2} \frac{\hat{a}_{2}(X)}{\sigma^{2}(X)}\right) \mid \mathbb{D}\right]
\end{aligned}
$$

and

$$
\begin{aligned}
\mathfrak{f}_{n, t, \hat{a}_{1}, \hat{a}_{2}}^{--}(x, z)= & \mathbf{1}\left[z \leq t\left\{1-n^{-1 / 2} u_{n}\right\}-n^{-1 / 2} v_{n}+\left\{1-t \frac{r(x)}{\sigma(x)}\right\} \frac{\hat{a}_{1}(x)}{\sigma(x)}+\frac{t}{2} \frac{\hat{a}_{2}(x)}{\sigma^{2}(x)}\right] \\
& -E\left[F\left(t\left\{1-n^{-1 / 2} u_{n}\right\}-n^{-1 / 2} v_{n}+\left\{1-t \frac{r(X)}{\sigma(X)}\right\} \frac{\hat{a}_{1}(X)}{\sigma(X)}+\frac{t}{2} \frac{\hat{a}_{2}(X)}{\sigma^{2}(X)}\right) \mid \mathbb{D}\right] .
\end{aligned}
$$

The remaining two sequences are defined similarly to those above. We will now consider the case $t \in[0, \infty)$. On this region, we can bound (4.3) by a sum of three terms:

$$
\begin{gathered}
\sup _{t \in[0, \infty)}\left|\frac{1}{n} \sum_{j=1}^{n}\left\{\mathfrak{f}_{n, t, \hat{a}_{1}, \hat{a}_{2}}^{++}\left(X_{j}, e_{j}\right)-\mathfrak{f}_{n, t, \hat{a}_{1}, \hat{a}_{2}}^{--}\left(X_{j}, e_{j}\right)\right\}\right| \\
\sup _{t \in[0, \infty)} \mid E\left[F\left(t\left\{1+n^{-1 / 2} u_{n}\right\}+n^{-1 / 2} v_{n}+\left\{1-t \frac{r(X)}{\sigma(X)}\right\} \frac{\hat{a}_{1}(X)}{\sigma(X)}+\frac{t}{2} \frac{\hat{a}_{2}(X)}{\sigma^{2}(X)}\right) \mid \mathbb{D}\right] \\
-E\left[F\left(t\left\{1-n^{-1 / 2} u_{n}\right\}-n^{-1 / 2} v_{n}+\left\{1-t \frac{r(X)}{\sigma(X)}\right\} \frac{\hat{a}_{1}(X)}{\sigma(X)}+\frac{t}{2} \frac{\hat{a}_{2}(X)}{\sigma^{2}(X)}\right) \mid \mathbb{D}\right] \mid
\end{gathered}
$$

and

$$
\begin{aligned}
\sup _{t \in[0, \infty)} \mid E[ & F\left(t+\frac{\hat{r}(X)-r(X)}{\sigma(X)}+t \frac{\hat{\sigma}(X)-\sigma(X)}{\sigma(X)}\right) \\
& \left.-F\left(t+\left\{1-t \frac{r(X)}{\sigma(X)}\right\} \frac{\hat{a}_{1}(X)}{\sigma(X)}+\frac{t}{2} \frac{\hat{a}_{2}(X)}{\sigma^{2}(X)}\right) \mid \mathbb{D}\right] \mid .
\end{aligned}
$$


Analogous arguments for the case of $t \in(-\infty, 0)$ lead to a similar bound, where $\mathfrak{f}_{n, t, \hat{a}_{1}, \hat{a}_{2}}^{+}$ replaces $\mathfrak{f}_{n, t, \hat{a}_{1}, \hat{a}_{2}}^{++}, \mathfrak{f}_{n, t, \hat{a}_{1}, \hat{a}_{2}}^{+-}$replaces $\mathfrak{f}_{n, t, \hat{a}_{1}, \hat{a}_{2}}^{--}$(4.5) is appropriately adjusted and the supremum in each term is restricted to $(-\infty, 0)$.

Following the arguments above, we will now specialize the asymptotic equicontinuity condition above to show (4.4) is $o_{p}\left(n^{-1 / 2}\right)$. Repeating the calculations above for $\operatorname{Var}\left[\mathfrak{f}_{t, \hat{a}_{1}, \hat{a}_{2}}(X, e)-\right.$ $\left.\mathfrak{f}_{t, 0,0}(X, e) \mid \mathbb{D}\right]$, we find, now for $t \in \mathbb{R}$,

$$
\begin{aligned}
& \sup _{t \in \mathbb{R}} \operatorname{Var}\left[\mathfrak{f}_{n, t, \hat{a}_{1}, \hat{a}_{2}}^{++}(X, e)-\mathfrak{f}_{n, t, \hat{a}_{1}, \hat{a}_{2}}^{--}(X, e) \mid \mathbb{D}\right] \\
& \leq E\left[F \left(\operatorname { m a x } \left\{t\left(1+n^{-1 / 2} u_{n}\right)+n^{-1 / 2} v_{n}+\left\{1-t \frac{r(X)}{\sigma(X)}\right\} \frac{\hat{a}_{1}(X)}{\sigma(X)}+\frac{t}{2} \frac{\hat{a}_{2}(X)}{\sigma^{2}(X)},\right.\right.\right. \\
& \left.\left.t\left(1-n^{-1 / 2} u_{n}\right)-n^{-1 / 2} v_{n}+\left\{1-t \frac{r(X)}{\sigma(X)}\right\} \frac{\hat{a}_{1}(X)}{\sigma(X)}+\frac{t}{2} \frac{\hat{a}_{2}(X)}{\sigma^{2}(X)}\right\}\right) \\
& -F\left(\operatorname { m i n } \left\{t\left(1+n^{-1 / 2} u_{n}\right)+n^{-1 / 2} v_{n}+\left\{1-t \frac{r(X)}{\sigma(X)}\right\} \frac{\hat{a}_{1}(X)}{\sigma(X)}+\frac{t}{2} \frac{\hat{a}_{2}(X)}{\sigma^{2}(X)},\right.\right. \\
& \left.\left.\left.t\left(1-n^{-1 / 2} u_{n}\right)-n^{-1 / 2} v_{n}+\left\{1-t \frac{r(X)}{\sigma(X)}\right\} \frac{\hat{a}_{1}(X)}{\sigma(X)}+\frac{t}{2} \frac{\hat{a}_{2}(X)}{\sigma^{2}(X)}\right\}\right) \mid \mathbb{D}\right] \\
& \leq 2 n^{-1 / 2} v_{n} \sup _{t \in \mathbb{R}} f(t)+2 n^{-1 / 2} u_{n} \sup _{t \in \mathbb{R}}|t f(t)| .
\end{aligned}
$$

This bound is $o_{p}\left(n^{-1 / 2}\right)$ and, therefore, $o_{p}(1)$, which implies the variance is asymptotically negligible: $\operatorname{Var}\left[\mathfrak{f}_{n, t, \hat{a}_{1}, \hat{a}_{2}}^{++}(X, e)-\mathfrak{f}_{n, t, \hat{a}_{1}, \hat{a}_{2}}^{--}(X, e) \mid \mathbb{D}\right]=o_{p}(1)$, uniformly in $t \in \mathbb{R}$. Hence, we have asymptotic equicontinuity and it follows for (4.4) to be $o_{p}\left(n^{-1 / 2}\right)$ as desired by further restricting $t$ to $[0, \infty)$. Continuing, we have

$$
\begin{aligned}
& \sup _{t \in \mathbb{R}} \mid E[ F\left(t\left(1+n^{-1 / 2} u_{n}\right)+n^{-1 / 2} v_{n}+\left\{1-t \frac{r(X)}{\sigma(X)}\right\} \frac{\hat{a}_{1}(X)}{\sigma(X)}+\frac{t}{2} \frac{\hat{a}_{2}(X)}{\sigma^{2}(X)}\right) \\
&\left.\quad-F\left(t\left(1-n^{-1 / 2} u_{n}\right)-n^{-1 / 2} v_{n}+\left\{1-t \frac{r(X)}{\sigma(X)}\right\} \frac{\hat{a}_{1}(X)}{\sigma(X)}+\frac{t}{2} \frac{\hat{a}_{2}(X)}{\sigma^{2}(X)}\right) \mid \mathbb{D}\right] \mid \\
& \leq 2 n^{-1 / 2} v_{n} \sup _{t \in \mathbb{R}} f(t)+2 n^{-1 / 2} u_{n} \sup _{t \in \mathbb{R}}|t f(t)| .
\end{aligned}
$$

Since $\left\{u_{n}\right\}_{n \geq 1}$ and $\left\{v_{n}\right\}_{n \geq 1}$ are both $o_{p}(1)$, it follows for the bound above to be $o_{p}\left(n^{-1 / 2}\right)$. This also implies (4.5) is $o_{p}\left(n^{-1 / 2}\right)$. Now using the identity for $t+\{\hat{r}(x)-r(x)\} / \sigma(x)+t\{\hat{\sigma}(x)-$ $\sigma(x)\} / \sigma(x)$, we have

$$
\begin{aligned}
& \sup _{t \in \mathbb{R}} \mid E\left[F\left(t+\frac{\hat{r}(X)-r(X)}{\sigma(X)}+t \frac{\hat{\sigma}(X)-\sigma(X)}{\sigma(X)}\right)\right. \\
&\left.-F\left(t+\left\{1-t \frac{r(X)}{\sigma(X)}\right\} \frac{\hat{a}_{1}(X)}{\sigma(X)}+\frac{t}{2} \frac{\hat{a}_{2}(X)}{\sigma^{2}(X)}\right) \mid \mathbb{D}\right] \mid \\
&=\sup _{t \in \mathbb{R}} \mid E[F(t+\left\{1-t \frac{r(X)}{\sigma(X)}\right\} \frac{\hat{a}_{1}(X)}{\sigma(X)}+\frac{t}{2} \frac{\hat{a}_{2}(X)}{\sigma^{2}(X)}+\frac{\hat{r}(X)-r(X)-\hat{a}_{1}(X)}{\sigma(X)} \\
&+t\left\{\frac{\hat{r}_{2}(X)-r_{2}(X)-\hat{a}_{2}(X)}{2 \sigma^{2}(X)}-\frac{r(X)}{\sigma(X)} \frac{\hat{r}(X)-r(X)-\hat{a}_{1}(X)}{\sigma(X)}\right. \\
&-\left.\left.-\frac{\{\hat{r}(X)-r(X)\}^{2}}{2 \sigma^{2}(X)}-\frac{\{\hat{\sigma}(X)-\sigma(X)\}^{2}}{2 \sigma^{2}(X)}\right\}\right) \\
&-\left.\left(t+\left\{1-\frac{r(X)}{\sigma(X)}\right\} \frac{\hat{a}_{1}(X)}{\sigma(X)}+\frac{t}{2} \frac{\hat{a}_{2}(X)}{\sigma^{2}(X)}\right) \mid \mathbb{D}\right] \mid \\
& \leq\left(\sup _{t \in \mathbb{R}} f(t)\left[\inf _{x \in[0,1]^{m}} \sigma(x)\right]^{-1}+\sup _{t \in \mathbb{R}}|t f(t)|\left[\sup _{x \in[0,1]^{m}}|r(x)|\right]\left[\inf _{x \in[0,1]^{m}} \sigma(x)\right]^{-2}\right)
\end{aligned}
$$




$$
\begin{aligned}
& \times \sup _{x \in[0,1]^{m}}\left|\hat{r}(x)-r(x)-\hat{a}_{1}(x)\right| \\
& +\frac{1}{2} \sup _{t \in \mathbb{R}}|t f(t)|\left[\inf _{x \in[0,1]^{m}} \sigma(x)\right]^{-2} \sup _{x \in[0,1]^{m}}\left|\hat{r}_{2}(x)-r_{2}(x)-\hat{a}_{2}(x)\right| \\
& +\frac{1}{2} \sup _{t \in \mathbb{R}}|t f(t)|\left[\inf _{x \in[0,1]^{m}} \sigma(x)\right]^{-2}\left(\sup _{x \in[0,1]^{m}}\{\hat{r}(x)-r(x)\}^{2}+\sup _{x \in[0,1]^{m}}\{\hat{\sigma}(x)-\sigma(x)\}^{2}\right) .
\end{aligned}
$$

Using the results above, this bound is $o_{p}\left(n^{-1 / 2}\right)$, which implies (4.6) is $o_{p}\left(n^{-1 / 2}\right)$. Since the same logic can be applied when $t \in(-\infty, 0)$, now using the function sequences $\left\{\mathfrak{f}_{n, t, \hat{a}_{1}, \hat{a}_{2}}^{-+}\right\}_{n \geq 1}$ and $\left\{\mathfrak{f}_{n, t, \hat{a}_{1}, \hat{a}_{2}}^{+-}\right\}_{n \geq 1}$, the analogous remainder terms to (4.4), (4.5) and (4.6) are all $o_{p}\left(n^{-1 / 2}\right)$ as well. Combining these results shows $\sup _{t \in \mathbb{R}}\left|R_{1}(t)\right|=o_{p}\left(n^{-1 / 2}\right)$.

From Remark 1, the random functions $\hat{a}_{1}$ and $\hat{a}_{2}$ additionally satisfy

$$
\int_{[0,1]^{m}}\left|\hat{a}_{1}(x)\right|^{1+b} g(x) d x=o_{p}\left(n^{-1 / 2}\right) \quad \text { and } \quad \int_{[0,1]^{m}}\left|\hat{a}_{2}(x)\right|^{1+b} g(x) d x=o_{p}\left(n^{-1 / 2}\right),
$$

where $b>m /(2 s-m)$. Setting $A(x)=\{\hat{r}(x)-r(x)\} / \sigma(x)$ and $B(x)=\{\hat{\sigma}(x)-\sigma(x)\} / \sigma(x)$, we can then bound $\sup _{t \in \mathbb{R}}\left|R_{2}(t)\right|$ by a sum of three terms:

$$
\begin{gathered}
\sup _{t \in \mathbb{R}}|E[F(t+A(X)+B(X) t)-F(t+B(X) t)-A(X) f(t+B(X) t) \mid \mathbb{D}]|, \\
\sup _{t \in \mathbb{R}}|E[F(t+B(X) t)-F(t)-B(X) t f(t) \mid \mathbb{D}]|
\end{gathered}
$$

and

$$
\sup _{t \in \mathbb{R}}|E[A(X)\{f(t+B(X) t)-f(t)\} \mid \mathbb{D}]| .
$$

To continue, we will require an additional result. Setting $x=\min \{t, t+B(X) t\}$ and $y=$ $\max \{t, t+B(X) t\}$, we have $0 \leq y-x=\max \{-B(X) t, B(X) t\}=|B(X) t|$, and we find, almost surely,

$$
\begin{aligned}
& 1 \\
\leq & \left.x^{2}\right)|f(y)-f(x)| \\
\leq & \left|\left(y^{2}\right) f(y)-\left(1+x^{2}\right) f(x)\right| \\
= & \mid \int_{x}^{y}\left\{\left(1+v^{2}\right) f^{\prime}(v) d v+\int_{x}^{y} 2 v f(v) d v \mid\right. \\
\leq & \int_{x}^{y}\left(1+v^{2}\right)^{1 / 2} f^{1 / 2}(v)\left(1+v^{2}\right)^{1 / 2}\left|\frac{f^{\prime}(v) \mid}{f(v)}\right| f^{1 / 2}(v) d v+2 \int_{x}^{y}|v f(v)| d v \\
\leq & \left\{\int_{x}^{x+y-x}\left(1+v^{2}\right) f(v) d v\right\}^{1 / 2}\left\{\int_{x}^{x+y-x}\left(1+v^{2}\right)\left(\frac{f^{\prime}(v)}{f(v)}\right)^{2} f(v) d v\right\}^{1 / 2} \\
& +2 \int_{x}^{x+y-x}|v f(v)| d v \\
\leq & \left\{\int_{0}^{1}\left(1+\{x+s(y-x)\}^{2}\right) f(x+s(y-x)) d s\right\}^{1 / 2} \\
& \times\left\{\int_{0}^{1}\left(1+\{x+s(y-x)\}^{2}\right)\left(\frac{f^{\prime}(x+s(y-x))}{f(x+s(y-x))}\right)^{2} f(x+s(y-x)) d s\right\}^{1 / 2} \times|B(X) t| \\
& +2\left(\sup _{t \in \mathbb{R}}|t f(t)|\right)|B(X) t| \\
\leq & \left(\left\{2 \int_{-\infty}^{\infty}\left(1+v^{2}\right)\left(\frac{f^{\prime}(v)}{f(v)}\right)^{2} F(d v)\right\}^{1 / 2}+2 \underset{t \in \mathbb{R}}{ }|t f(t)|\right)|B(X) t| .
\end{aligned}
$$


This implies

$$
\begin{aligned}
& |f(t+B(X) t)-f(t)| \\
& =|f(\max \{t, t+B(X) t\})-f(\min \{t, t+B(X) t\})| \\
& \leq\left(\left\{2 \int_{-\infty}^{\infty}\left(1+v^{2}\right)\left(\frac{f^{\prime}(v)}{f(v)}\right)^{2} F(d v)\right\}^{1 / 2}+2 \sup _{t \in \mathbb{R}}|t f(t)|\right) \frac{|t|}{1+t^{2} \min \left\{1,(1+B(X))^{2}\right\}}|B(X)| \\
& \leq\left(\left\{2 \int_{-\infty}^{\infty}\left(1+v^{2}\right)\left(\frac{f^{\prime}(v)}{f(v)}\right)^{2} F(d v)\right\}^{1 / 2}+2 \sup _{t \in \mathbb{R}}|t f(t)|\right) \frac{|t|}{1+t^{2} \min \left\{1,(1+L)^{2}\right\}}|B(X)|,
\end{aligned}
$$

almost surely, where $L=\inf _{x \in[0,1]^{m}} B(x)$. We have already shown that $\sup _{x \in[0,1]^{m}}|B(x)|=o(1)$ above, almost surely, and, for large enough $n,-1<\inf _{x \in[0,1]^{m}} B(x)=L$. Hence, for large enough $n$, the bound above is finite.

It then follows for the map $t \mapsto f(t)$ to be Hölder with exponent $b$. Writing $K_{f, b}$ for the Hölder constant for $f$ with exponent $b$, we have

$$
\begin{aligned}
& \sup _{t \in \mathbb{R}}|E[F(t+A(X)+B(X) t)-F(t+B(X) t)-A(X) f(t+B(X) t) \mid \mathbb{D}]| \\
& \leq \sup _{t \in \mathbb{R}} E\left[|A(X)| \int_{0}^{1}|f(t+s A(X)+B(X) t)-f(t+B(X) t)| d s \mid \mathbb{D}\right] \\
& \leq K_{f, b} \int_{0}^{1}|s|^{b} d s E\left[|A(X)|^{1+b} \mid \mathbb{D}\right] \\
& =C_{1} E\left[|A(X)|^{1+b} \mid \mathbb{D}\right],
\end{aligned}
$$

choosing $C_{1}=K_{f, b} /\{1+b\}$. Observing that for any real numbers $x$ and $y$, and $0<c$, that $|x+y|^{1+c} \leq 2^{c}\left(|x|^{1+c}+|y|^{1+c}\right)$, we find

$$
\begin{aligned}
& E\left[|A(X)|^{1+b} \mid \mathbb{D}\right] \\
& =E\left[\left|\frac{\hat{a}_{1}(X)}{\sigma(X)}+\frac{\hat{r}(X)-r(x)-\hat{a}_{1}(X)}{\sigma(X)}\right|^{1+b} \mid \mathbb{D}\right] \\
& \leq 2^{b}\left[\inf _{x \in[0,1]^{m}} \sigma(x)\right]^{1+b}\left(\int_{[0,1]^{m}}\left|\hat{a}_{1}(x)\right|^{1+b} g(x) d x+\left[\sup _{x \in[0,1]^{m}}\left|\hat{r}(x)-r(x)-\hat{a}_{1}(x)\right|\right]^{1+b}\right) .
\end{aligned}
$$

Using the results above, this bound is $o_{p}\left(n^{-1 / 2}\right)$, and, therefore, (4.8) is $o_{p}\left(n^{-1 / 2}\right)$.

Using the same procedure as above, we find

$$
\begin{aligned}
& \sup _{t \in \mathbb{R}}|E[F(t+B(X) t)-F(t)-B(X) t f(t) \mid \mathbb{D}]| \\
&=\sup _{t \in \mathbb{R}}\left|E\left[B(X) t \int_{0}^{1}\{f(t+s B(X) t)-f(t)\} d s \mid \mathbb{D}\right]\right| \\
& \leq \sup _{t \in \mathbb{R}} E\left[|B(X)| \int_{0}^{1}|t||f(t+s B(X) t)-f(t)| d s \mid \mathbb{D}\right] \\
& \leq\left(\left\{2 \int_{-\infty}^{\infty}\left(1+v^{2}\right)\left(\frac{f^{\prime}(v)}{f(v)}\right)^{2} F(d v)\right\}^{1 / 2}+2 \sup _{t \in \mathbb{R}}|t f(t)|\right) \\
& \times\left(\sup _{t \in \mathbb{R}} \sup _{s \in[0,1]} \frac{s t^{2}}{1+t^{2} \min \left\{1,(1+s L)^{2}\right\}}\right) \times E\left[B^{2}(X) \mid \mathbb{D}\right] \\
& \leq\left(\left\{2 \int_{-\infty}^{\infty}\left(1+v^{2}\right)\left(\frac{f^{\prime}(v)}{f(v)}\right)^{2} F(d v)\right\}^{1 / 2}+2 \sup _{t \in \mathbb{R}}|t f(t)|\right) \\
& \times\left(\sup _{t \in \mathbb{R}} \frac{t^{2}}{1+t^{2} \min \left\{1,(1+L)^{2}\right\}}\right) E\left[B^{2}(X) \mid \mathbb{D}\right]
\end{aligned}
$$




$$
\leq C_{2} E\left[B^{2}(X) \mid \mathbb{D}\right]
$$

for large enough $n$, choosing $C_{2}$ proportional to

$$
\left(\left\{2 \int_{-\infty}^{\infty}\left(1+v^{2}\right)\left(\frac{f^{\prime}(v)}{f(v)}\right)^{2} F(d v)\right\}^{1 / 2}+2 \sup _{t \in \mathbb{R}}|t f(t)|\right)\left(\sup _{t \in \mathbb{R}} \frac{t^{2}}{1+t^{2} \min \left\{1,(1+L)^{2}\right\}}\right)
$$

Continuing, $E\left[B^{2}(X) \mid \mathbb{D}\right]$ is equal to

$$
\begin{aligned}
& E\left[\left(\frac{\hat{a}_{2}(X)}{2 \sigma^{2}(X)}-\frac{r(X)}{\sigma(X)} \frac{\hat{a}_{1}(X)}{\sigma(X)}+\frac{\hat{r}_{2}(X)-r_{2}(X)-\hat{a}_{2}(X)}{2 \sigma^{2}(X)}-\frac{r(X)}{\sigma(X)} \frac{\hat{r}(X)-r(X)-\hat{a}_{1}(X)}{\sigma(X)}\right.\right. \\
& \left.\left.\quad-\frac{\{\hat{r}(X)-r(X)\}^{2}}{2 \sigma^{2}(X)}-\frac{\{\hat{\sigma}(X)-\sigma(X)\}^{2}}{2 \sigma^{2}(X)}\right)^{2} \mid \mathbb{D}\right],
\end{aligned}
$$

which is bounded by

$$
\begin{aligned}
& {\left[\inf _{x \in[0,1]^{m}} \sigma(x)\right]^{-4}\left(\frac{1}{2} \int_{[0,1]^{m}}\left|\hat{a}_{2}(x)\right|^{2} g(x) d x+2\left[\sup _{x \in[0,1]^{m}}\left|\hat{r}_{2}(x)-r_{2}(x)-\hat{a}_{2}(x)\right|\right]^{2}\right.} \\
& +4\left[\sup _{x \in[0,1]^{m}}|r(x)|\right]^{2} \int_{[0,1]^{m}}\left|\hat{a}_{1}(x)\right|^{2} g(x) d x+8\left[\sup _{x \in[0,1]^{m}}\{\hat{r}(x)-r(x)\}^{2}\right]^{2} \\
& \left.+8\left[\sup _{x \in[0,1]^{m}}\{\hat{\sigma}(x)-\sigma(x)\}^{2}\right]^{2}+16\left[\sup _{x \in[0,1]^{m}}|r(x)|\right]^{2}\left[\sup _{x \in[0,1]^{m}}\left|\hat{r}(x)-r(x)-\hat{a}_{1}(x)\right|\right]^{2}\right) .
\end{aligned}
$$

It then follows from the results above and (4.7) for this bound to be $o_{p}\left(n^{-1 / 2}\right)$. This implies (4.9) is $o_{p}\left(n^{-1 / 2}\right)$.

Again, using the procedure above, we find

$$
\begin{aligned}
\sup _{t \in \mathbb{R}}|E[A(X)\{f(t+B(X) t)-f(t)\} \mid \mathbb{D}]| \\
\leq \sup _{t \in \mathbb{R}} E[|A(X)||f(t+B(X) t)-f(t)| \mid \mathbb{D}] \\
\leq\left(\left\{2 \int_{-\infty}^{\infty}\left(1+v^{2}\right)\left(\frac{f^{\prime}(v)}{f(v)}\right)^{2} F(d v)\right\}^{1 / 2}+2 \sup _{t \in \mathbb{R}}|t f(t)|\right) \\
\quad \times\left(\sup _{t \in \mathbb{R}} \frac{|t|}{1+t^{2} \min \left\{1,(1+L)^{2}\right\}}\right) E[|A(X) B(X)| \mid \mathbb{D}] \\
\leq C_{3} E[|A(X) B(X)| \mid \mathbb{D}],
\end{aligned}
$$

for large enough $n$, choosing $C_{3}$ proportional to

$$
\left(\left\{2 \int_{-\infty}^{\infty}\left(1+v^{2}\right)\left(\frac{f^{\prime}(v)}{f(v)}\right)^{2} F(d v)\right\}^{1 / 2}+2 \sup _{t \in \mathbb{R}}|t f(t)|\right)\left(\sup _{t \in \mathbb{R}} \frac{|t|}{1+t^{2} \min \left\{1,(1+L)^{2}\right\}}\right) .
$$

Combining the calculations above, we have

$$
\begin{aligned}
E & {[|A(X) B(X)| \mid \mathbb{D}] } \\
\leq & E^{1 / 2}\left[|A(X)|^{2} \mid \mathbb{D}\right] E^{1 / 2}\left[|B(X)|^{2} \mid \mathbb{D}\right] \\
\leq & \sqrt{2}\left[\inf _{x \in[0,1]^{m}} \sigma(x)\right]^{3}\left(\int_{[0,1]^{m}}\left|\hat{a}_{1}(x)\right|^{2} g(x) d x+\left[\sup _{x \in[0,1]^{m}}\left|\hat{r}(x)-r(x)-\hat{a}_{1}(x)\right|\right]^{2}\right)^{1 / 2} \\
& \times\left(\frac{1}{2} \int_{[0,1]^{m}}\left|\hat{a}_{2}(x)\right|^{2} g(x) d x+4\left[\sup _{x \in[0,1]^{m}}|r(x)|\right]^{2} \int_{[0,1]^{m}}\left|\hat{a}_{1}(x)\right|^{2} g(x) d x\right.
\end{aligned}
$$




$$
\begin{aligned}
& +2\left[\sup _{x \in[0,1]^{m}}\left|\hat{r}_{2}(x)-r_{2}(x)-\hat{a}_{2}(x)\right|\right]^{2} \\
& +8\left[\sup _{x \in[0,1]^{m}}\{\hat{r}(x)-r(x)\}^{2}\right]^{2}+8\left[\sup _{x \in[0,1]^{m}}\{\hat{\sigma}(x)-\sigma(x)\}^{2}\right]^{2} \\
& \left.+16\left[\sup _{x \in[0,1]^{m}}|r(x)|\right]^{2}\left[\sup _{x \in[0,1]^{m}}\left|\hat{r}(x)-r(x)-\hat{a}_{1}(x)\right|\right]^{2}\right)^{1 / 2} .
\end{aligned}
$$

Again, it follows from the results above and (4.7) for this bound to be $o_{p}\left(n^{-1 / 2}\right)$, which implies (4.10) is $o_{p}\left(n^{-1 / 2}\right)$. Combining the above results for (4.8), (4.9) and (4.10) shows that $\sup _{t \in \mathbb{R}}\left|R_{2}(t)\right|=o_{p}\left(n^{-1 / 2}\right)$.

It follows from the discussion in Remark 1 for analogous conclusions of Proposition 1 to hold for the estimators $\hat{r}$ and $\hat{\sigma}$, i.e.

$$
\left|\int_{[0,1]^{m}} \frac{\hat{r}(x)-r(x)}{\sigma(x)} g(x) d x-\frac{1}{n} \sum_{j=1}^{n} e_{j}\right|=o_{p}\left(n^{-1 / 2}\right)
$$

and

$$
\left|\int_{[0,1]^{m}} \frac{\hat{\sigma}(x)-\sigma(x)}{\sigma(x)} g(x) d x-\frac{1}{n} \sum_{j=1}^{n} \frac{e_{j}^{2}-1}{2}\right|=o_{p}\left(n^{-1 / 2}\right) .
$$

Since we have $\sup _{t \in \mathbb{R}} f(t)<\infty$ and $\sup _{t \in \mathbb{R}}|t f(t)|<\infty$, we find $\sup _{t \in \mathbb{R}}\left|R_{3}(t)\right|=o_{p}\left(n^{-1 / 2}\right)$. This concludes the proof of Theorem 1 .

\section{Acknowledgements}

Justin Chown gratefully acknowledges financial support from the contract 'Projet d'Actions de Recherche Concertées' (ARC) 11/16-039 of the 'Communauté française de Belgique', granted by the 'Académie universitaire Louvain', and the Collaborative Research Center "Statistical modeling of nonlinear dynamic processes" (SFB 823, Teilprojekt C4) of the German Research Foundation (DFG). I would like to thank the two referees for their careful reading of the manuscript and the very helpful comments that greatly improved the quality of this work.

\section{References}

[1] Asteriou, D. and Hall, S.G. (2011). Applied Econometrics. Palgrave MacMillan, New York, New York. ISBN 9780230271821.

[2] Chown, J. and Müller, U.U. (2013). Efficiently estimating the error distribution in nonparametric regression with responses missing at random. J. Nonparametr. Stat. 25, 665-677.

[3] Greene, W.H. (2000), Econometric Analysis, Prentice Hall, Upper Saddle River, New Jersey. ISBN 9780130132970.

[4] Koul, H.L., Müller, U.U. and Schick, A. (2012). The transfer principle: a tool for complete case analysis. Ann. Statist. 40, 3031-3049.

[5] Little, R.J.A. and Rubin, D.B. (2002). Statistical analysis with missing data. Wiley series in probability and mathematical statistics. Wiley, Hoboken, New Jersey. ISBN 9780471183860.

[6] Masry, E. (1996). Multivariate local polynomial regression for time series: uniform strong consistency rates. J. Time Series Anal. 17, 571-599.

[7] Müller, U.U., Schick, A. and Wefelmeyer, W. (2004). Estimating linear functionals of the error distribution in nonparametric regression. J. Statist. Plann. Inference 119, 75-93.

[8] Müller, U.U., Schick, A. and Wefelmeyer, W. (2006). Imputing responses that are not missing. In Probability, Statistics and Modelling in Public Health, eds. Nikulin, M., Commenges, D. and Huber, C., pages 350-363. Springer, New York, New York. ISBN 9780387260235.

[9] Müller, U.U., Schick A. and Wefelmeyer W. (2007). Estimating the error distribution in semiparametric regression. Statist. Decisions 25, 1-18.

[10] Müller, U.U., Schick, A. and Wefelmeyer, W. (2009). Estimating the error distribution function in nonparametric regression with multivariate covariates. Statist. Probab. Lett. 79, 957-964. 
[11] Neumeyer, N. and Van Keilegom, I. (2010). Estimating the error distribution in nonparametric multiple regression with applications to model testing. J. Multivariate Anal. 101, 1067-1078.

[12] Schick, A. (1993). On efficient estimation in regression models. Ann. Statist. 21, 1486-1521.

[13] Schick, A. (1994). On efficient estimation in regression models with unknown scale functions. Math. Methods Statist. 3, 171-212.

[14] Sheather, S.J. (2009). A Modern Approach to Regression with R. Springer Series in Language and Communication. Springer, New York, New York. ISBN 9780387096087.

[15] Van der Vaart, A.W. and Wellner, J.A. (1996). Weak convergence and empirical processes. With applications to statistics. Springer Series in Statistics. Springer-Verlag, New York. ISBN 9781475725476.

[16] Vinod, H.D. (2008). Hands-on Intermediate Econometrics Using R: Templates for Extending Dozens of Practical Examples. World Scientific, Hackensack, New Jersey. ISBN 9789812818850. 